\title{
Effects of Tip Clearance Gap and Exit Mach Number on Turbine Blade Tip and Near-Tip Heat Transfer
}

\author{
Karu Anto \\ Thesis submitted to the faculty of the \\ Virginia Polytechnic Institute and State University \\ in partial fulfillment of the requirements for the degree of \\ Master of Science \\ In \\ Mechanical Engineering
}

Wing F. Ng, Chair

Srinath V. Ekkad

Thomas E. Diller

May 2, 2012

Blacksburg, Virginia

Keywords: Heat Transfer, Tip Clearance, Turbine Blade, Thin Film Gage, Transonic 


\title{
Effects of Tip Clearance Gap and Exit Mach Number on Turbine Blade Tip and Near-Tip Heat Transfer
}

\author{
Karu Anto \\ Abstract \\ It is important to be able to accurately predict the heat transfer distribution over a \\ component when designing the turbine section of an engine. The gas temperature exiting the \\ combustor in many gas turbine engines is above the melting temperature of turbine components. \\ These high gas temperatures can quickly degrade components and cause failure. A detailed heat \\ transfer study on these turbine components is an important input when developing ways to cool \\ them. One such important component is the gas turbine blade, which primarily extracts the \\ energy from the hot combustion gases. To allow for rotation of the blades, a gap exists between \\ the stationary casing and the rotating blades. Unfortunately, leakage flow from the blade pressure \\ side to the suction side dramatically increases the heat load on and around the blade tip surfaces. \\ The objective of this work is to present the heat transfer distribution on the tip and near- \\ tip region of a turbine blade under transonic conditions. Tests were performed at a high inlet \\ freestream turbulence level $(\mathrm{Tu}=12 \%)$. This work seeks to investigate the effects of tip \\ clearance gap and Mach number on heat transfer on and near the blade tip. Thin film heat flux \\ gages that allow for high frequency measurements are used to obtain the heat transfer \\ distribution on the near tip pressure and suction sides. An infrared thermography technique is \\ used to obtain the heat transfer distribution on the blade tip surface.
}




\section{Preface}

In order to get more energy from gas turbine engines, the temperature of the combustion products is continually being increased by designers. These high temperatures will lead to increased degradation of turbine blades unless effective cooling schemes are developed to help protect the blades. A thorough understanding of the heat transfer distribution on the gas turbine blades is essential before sound cooling techniques can be implemented. An especially fragile part of the turbine blade exposed to high heat transfer is the blade tip. The tip gap, between the casing and the blade tip, is where leakage flow escapes from the blade pressure side to the suction side. This leakage flow has been shown to significantly increase the heat load on the blade tip. Designers need to understand the heat transfer distribution, especially on the blade tip, and then subsequently devise the appropriate cooling mechanisms. This study examines the effects of tip clearance gap and exit Reynolds number on the heat transfer distribution on a turbine blade tip and near-tip region.

This thesis is organized into two sections that include one research paper that concisely documents the central thrust of this study. The author was primarily responsible for most aspects of the research project from initial test section design to analyzing data. A colleague, Xue Song, was responsible for the post processing of some of the blade tip heat transfer results. The title of the research paper coincides with the title of this thesis. A series of appendices then follow which provide additional information on several aspects of the present study including instrumentation, experimental setup, and heat transfer results. 


\section{Acknowledgements}

I would like to thank a whole host of people who have helped me in many ways as I seek to achieve my degree. My professors, family members, departmental staff and friends have all been instrumental in unique ways. I express my gratitude to God for his guidance, mercy and grace for allowing me work through gaining my Master's degree.

Firstly, I would like to extend my profound gratitude to my advisor Dr. Wing Ng for his professional mentorship and support throughout my graduate studies. His expertise in the field of turbomachinery was of tremendous value as he helped me navigate through advanced level research. I would also like to thank my committee member, Dr. Srinath Ekkad for all the help and support he provided when I came to him with questions on many occasions. I learned a lot on Heat Transfer directly under Dr. Ekkad. Many thanks to Dr. Thomas Diller, also my committee member, for spending time to review this research work. I am grateful to Dr. Hee Koo Moon and Dr. Luzeng Zhang of Solar Turbines Inc., our industry sponsors, for their continued support of our research group.

I couldn't have done this without the tremendous help of my colleagues and fellow graduate students. Xue Song has especially provided the much needed guidance that I needed from the onset and all through to the end. His experience with the transonic wind tunnel facility was tremendous in helping me safely use the facility. I am indeed very grateful. Great thanks to Arnab Roy who helped me conduct experiments especially on weekends and other after-office hours. Thanks to Colin Reagle and Jacob Delimont for providing both direct and indirect ideas throughout this project. I'll like to thank Patrick Seiler for providing some helping hands during experimentation early on in this work. Without the services of our technicians in the machine shop, we would not have been able to produce concrete parts from our conceptual designs. To this end, I would like to thank Johnny Cox and Bill Songer. Bill has had to cope with my regular visits to the shop for fabrication, and to help fix some irregularities that may have been left out in the initial design phase of some parts.

Finally, I would like to thank my parents, Solomon Anto and Alisabatu Anto for all their financial and emotional support throughout my education to this point. I am truly grateful for your impact in my life. I also thank my siblings, Matthias Anto, Lois Sajoh and Nympha Anto for their support. I am very grateful to my best friend Huseina Edili Ogaji for providing encouragement and unconditional support throughout this endeavor. 
All photographs in this work by author, 2012. 


\section{Recognition of Co-authors}

Song Xue is a PhD candidate with the turbomachinery group at the Virginia Tech Mechanical Engineering Department. His primary research focus is on gas turbine blade film cooling. Xue's contributions to this research work span from the initial design concepts employed in the test section through the data reduction techniques used to obtain heat transfer data. He was present at most team meetings and spearheaded major sections of this work to include conducting boundary layer measurements and reducing portions of the blade tip heat transfer data.

Wing F. Ng is the Chris Kraft Endowed Professor of Engineering at the Virginia Tech Mechanical Engineering Department. Ng's primary research focus centers on experimentally understanding the aerodynamic and heat transfer characteristics of several gas turbine engine components. He is the recipient of several teaching awards from the Virginia Tech College of Engineering and has co-authored a number of award winning papers at major Engineering conferences. In the present research, $\mathrm{Ng}$ was the primary advisor and mentor throughout the duration of this project. He coordinated research expenditures and provided technical guidance on the direction and goals of the project. 


\section{Table of Contents}

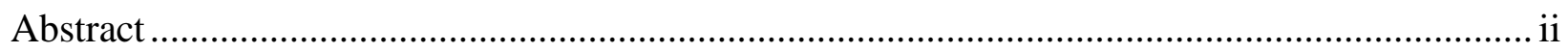

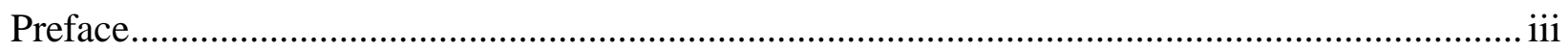

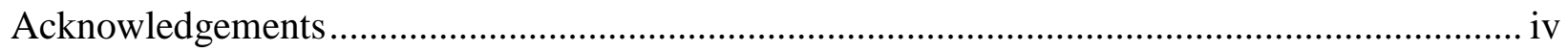

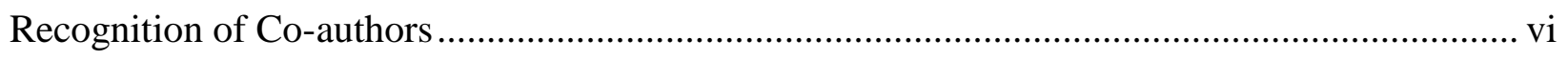

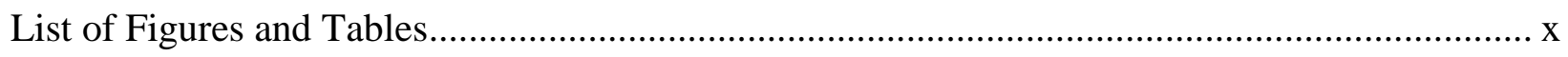

Effects of Tip Clearance Gap and Exit Mach Number on Turbine Blade Tip and Near-Tip Heat

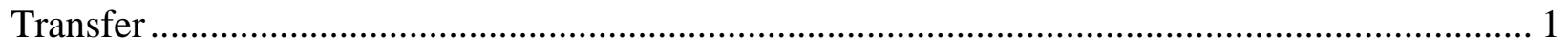

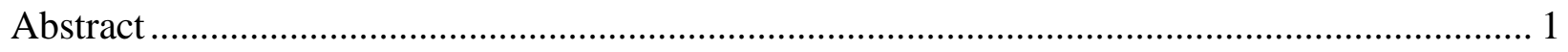

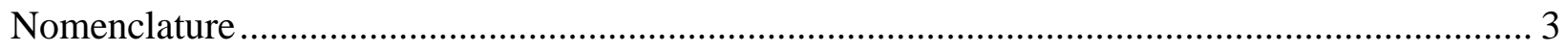

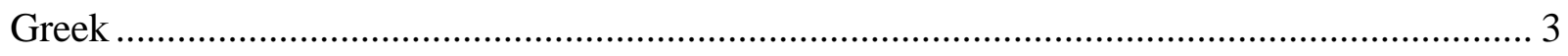

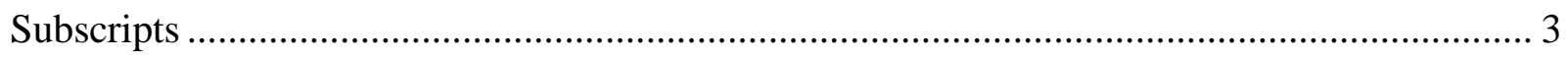

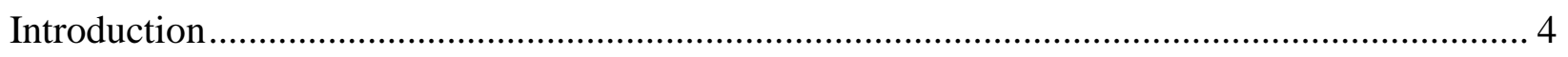

Summary of Past Literature ........................................................................................ 4

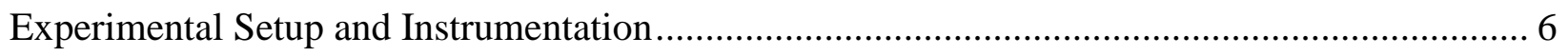

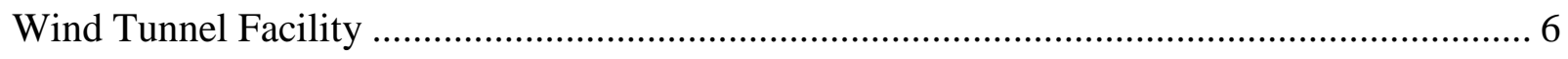

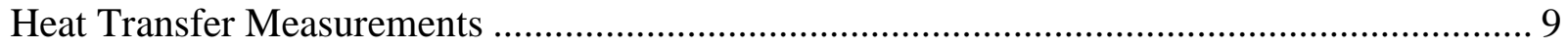

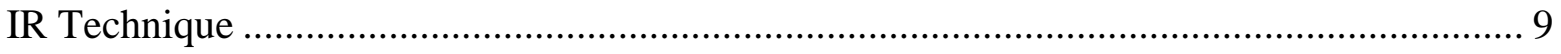

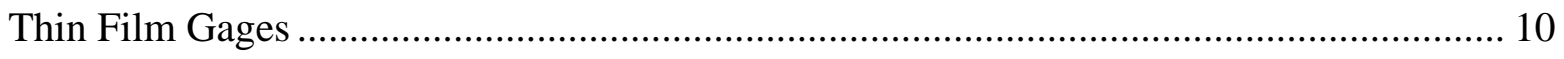

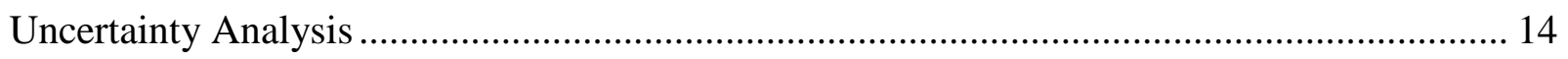

Blade Static Pressure Distribution at Midspan............................................................. 14

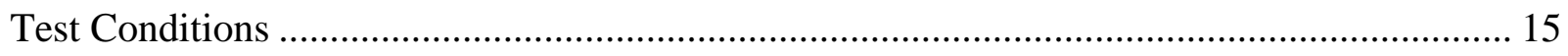

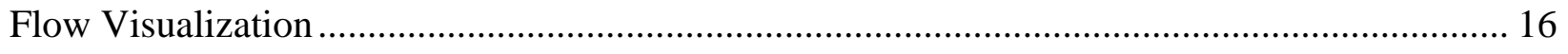

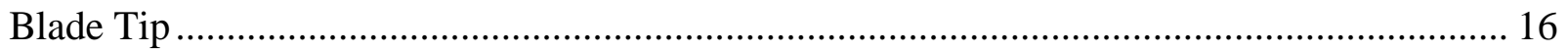




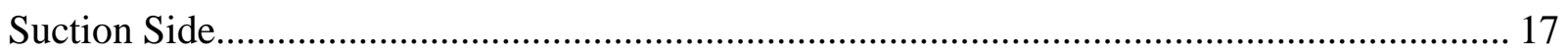

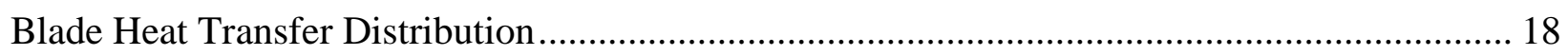

Heat Transfer on the Blade Tip ........................................................................................ 18

Averaged Heat Transfer Coefficient............................................................................... 20

Heat Transfer on the Near-Tip Region................................................................................ 22

Basic Heat Transfer Characteristics at the Design Condition ............................................. 22

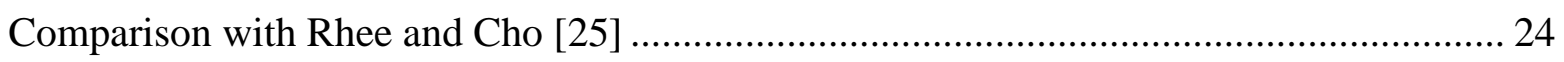

Exit Mach Number Effects on Heat Transfer Distribution................................................... 25

Tip Clearance Effects on Heat Transfer Distribution ............................................................ 26

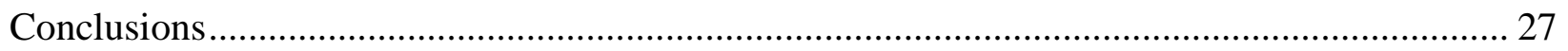

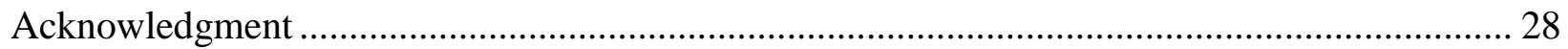

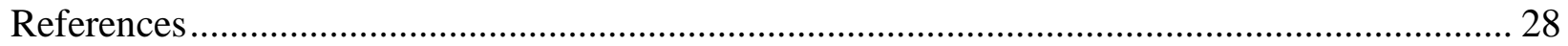

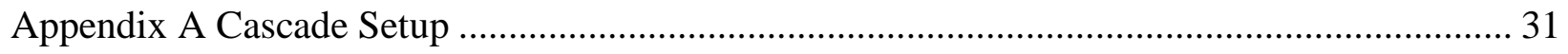

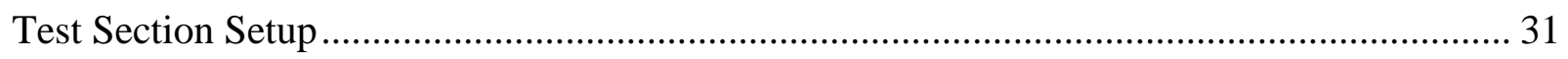

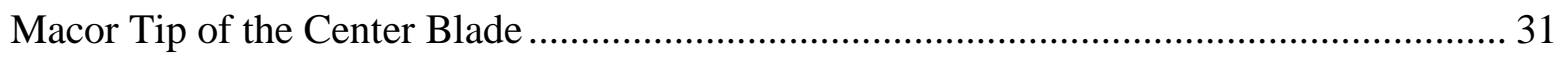

Mounting and Adjustment of Blade Tip Clearance in the Test Section................................. 31

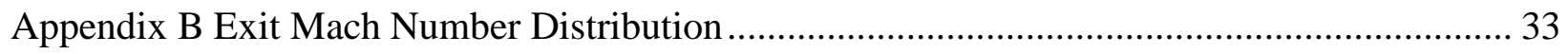

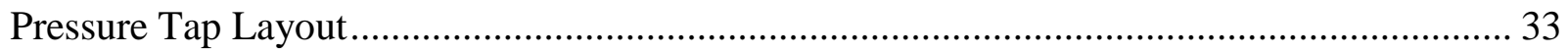

Static Pressure Measurement Technique ................................................................................... 33

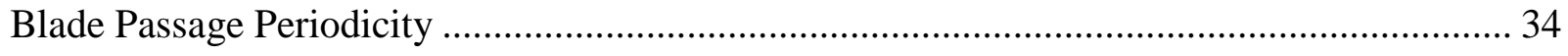

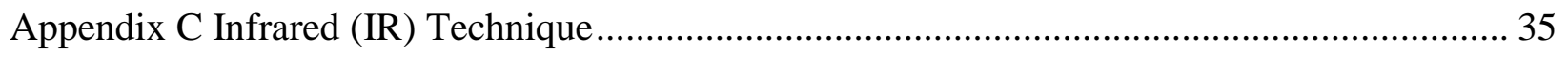

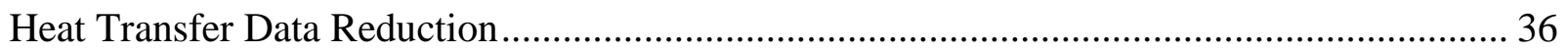




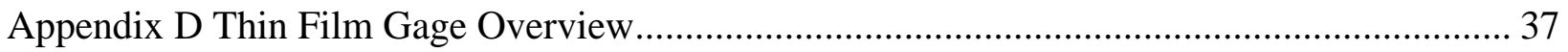

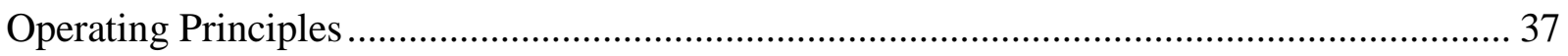

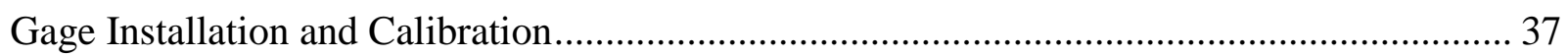

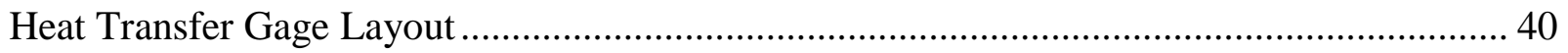

Thin Film Gauge Data Collection .......................................................................... 41

Heat Transfer Data Reduction................................................................................. 42

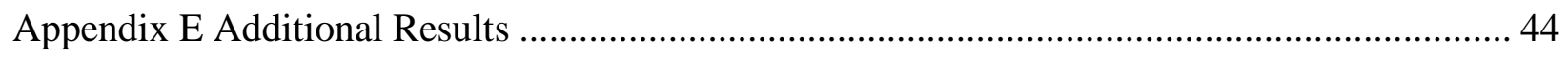

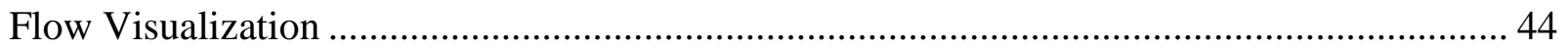

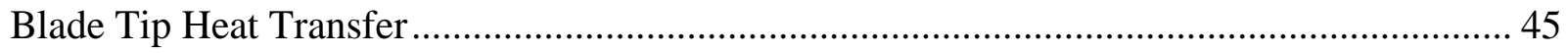

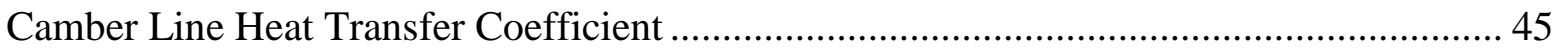

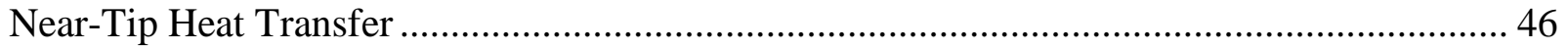

Tip Clearance Effects on Heat Transfer Distribution .................................................. 46

Exit Mach number Effects on Heat Transfer Distribution ......................................... 48

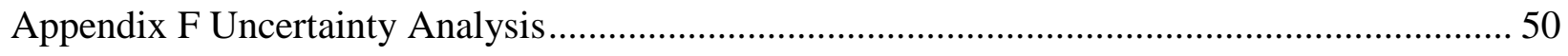

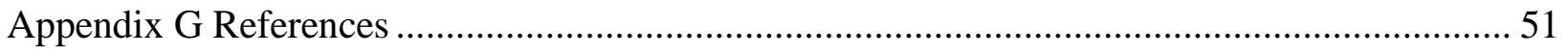




\section{List of Figures and Tables}

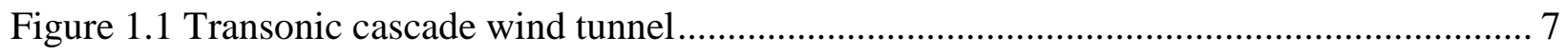

Figure 1.2 Cascade diagram showing the blades ............................................................... 8

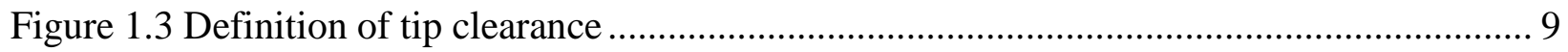

Figure 1.4 Blade profile showing thin film gage locations............................................... 10

Figure 1.5 Thin film gages in wind tunnel test section ................................................. 11

Figure 1.6 Inlet endwall boundary layer profile measured approximately $0.18 \mathrm{C}$ upstream of the

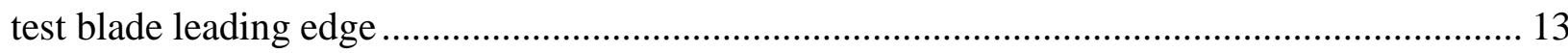

Figure 1.7 Typical mainstream and blade surface temperatures history ................................ 13

Figure 1.8 Local Mach number distribution over blade .................................................. 14

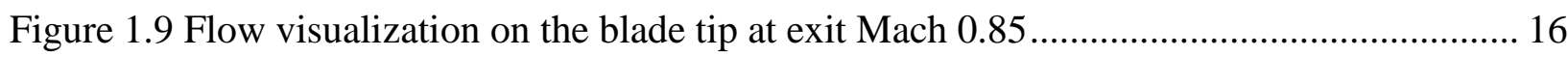

Figure 1.10 Suction side flow visualization at $\mathrm{M}=0.85$ with a tip clearance of (a) $0.9 \%$ (baseline)

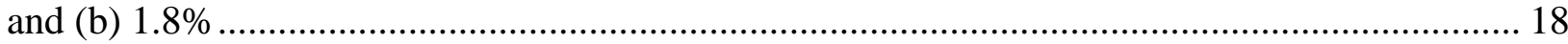

Figure 1.11 Heat transfer coefficient distribution on the blade tip at exit Mach 0.85 ............... 19

Figure 1.12 Heat transfer coefficient distribution on the blade tip at exit Mach 1.05 ............... 19

Figure 1.13 Circumferential averaged heat transfer on the blade tip at exit Mach $0.85 \ldots . . . . . . . . .21$

Figure 1.14 Circumferential averaged heat transfer on the blade tip for $0.9 \%$ tip gap.............. 21

Figure 1.15 Heat transfer distribution at the design condition with exit Mach 0.85, 0.9\% tip gap

Figure 1.16 Comparison of heat/mass transfer with Rhee et al. [25] ..................................... 24

Figure 1.17 Heat transfer distribution for $0.9 \%$ tip clearance ............................................. 25

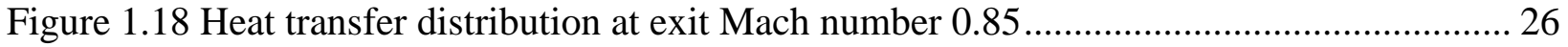

Figure A.1 Macor tip of the test blade ............................................................................. 31

Figure A.2 Mounting and adjustment of tip clearance in the test section................................. 32

Figure A.3 Picture of test section with instrumented blade in place .................................... 32

Figure B.1 Schematic showing the pressure tap locations on the endwall .............................. 33

Figure B.2 Local exit Mach comparison for a Mach 1 test ..................................................... 34 


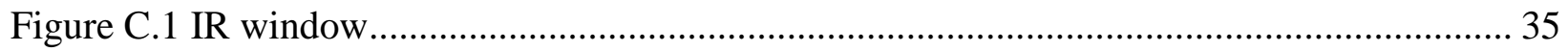

Figure C.2 Example curvefit of blade IR data ................................................................. 36

Figure D.1 Diagram of multi-layered gage design (not drawn to scale) ................................. 37

Figure D.2 Picture of test blade with thin film gage installed ............................................. 38

Figure D.3 Calibration of a single gage that exhibited unstable behavior ................................ 39

Figure D.4 Calibration of a single gage that exhibited stable behavior ................................... 39

Figure D.5 Schematic of the Wheatstone bridge circuit for the thin film gauges ..................... 40

Figure D.6 Blade profile showing thin film gage locations ................................................. 41

Figure D.7 Sample surface temperature and heat flux histories .......................................... 42

Figure D.8 Determination of heat transfer coefficient .................................................... 43

Figure E.1 Suction side flow visualization at $M=1.05$ with a tip clearance of $0.9 \% \ldots \ldots \ldots \ldots \ldots . . . .44$

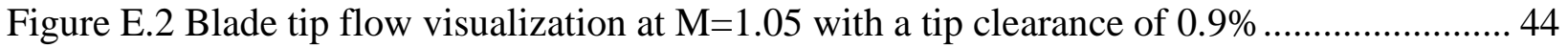
Figure E.3 Measured heat transfer coefficients along the camber line of the blade tip at exit Mach

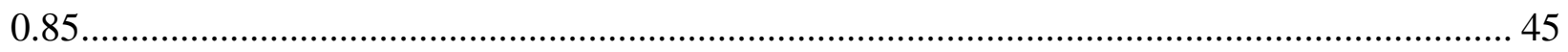

Figure E.4 Measured heat transfer coefficients along the camber line of the blade tip at $0.9 \%$ tip

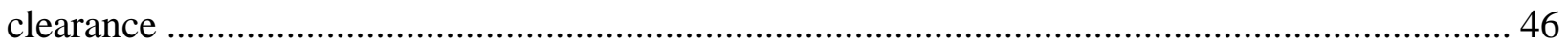

Figure E.5 Heat transfer distribution at exit Mach number 0.7 ............................................ 47

Figure E.6 Heat transfer distribution at exit Mach number 1.05 ............................................. 47

Figure E.7 Heat transfer distribution for 1.8\% tip clearance ............................................... 48

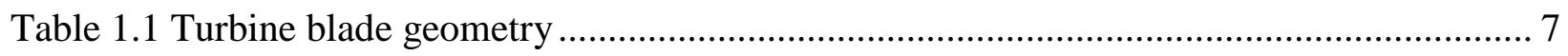

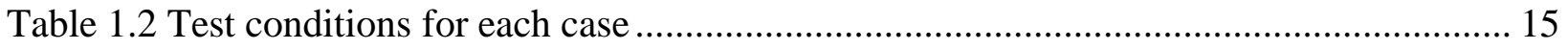




\title{
Effects of Tip Clearance Gap and Exit Mach Number on Turbine Blade Tip and Near-Tip Heat Transfer
}

\author{
K. Anto, S. Xue and W.F. Ng \\ Mechanical Engineering Department \\ Virginia Polytechnic Institute and State University \\ Blacksburg, VA 24061 \\ L.J. Zhang and H.K. Moon \\ Solar Turbines Inc. \\ San Diego, CA 92101
}

\section{Abstract}

The present study focuses on local heat transfer characteristics on the tip and near-tip regions of a turbine blade with a flat tip, tested under transonic conditions in a stationary, 2-D linear cascade. The experiments were conducted at the Virginia Tech transonic blow-down wind tunnel facility. The effects of tip clearance and exit Mach number on heat transfer distribution were investigated on the tip surface using a transient infrared thermography technique. In addition, thin film gages were used to study similar effects in heat transfer on the near-tip regions at $94 \%$ height based on engine blade span of the pressure and suction sides. Surface oil flow visualizations on the blade tip region were carried-out to shed some light on the leakage flow structure. Experiments were performed at three exit Mach numbers of 0.7, 0.85, and 1.05 for two different tip clearances of $0.9 \%$ and $1.8 \%$ based on engine blade span. The exit Mach numbers tested correspond to exit Reynolds numbers of $7.6 \times 10^{5}, 9.0 \times 10^{5}$, and $1.1 \times 10^{6}$ based on blade true chord. The tests were performed with a high freestream turbulence intensity of $12 \%$ at the cascade inlet.

Results at 0.85 exit Mach showed that an increase in the tip gap clearance translates into a $12 \%$ increase in the heat transfer coefficients on the blade tip surface. At $0.9 \%$ tip clearance, an increase in exit Mach number from 0.85 to 1.05 led to a $24 \%$ increase in heat transfer on the tip. High heat transfer was observed at the leading edge area of the blade tip, and an increase in the tip clearance gap and exit Mach number augmented this leading edge heat transfer. At $94 \%$ of engine blade height on the suction side near the tip, a peak in heat transfer was observed in all 
test cases at $\mathrm{s} / \mathrm{C}=0.66$ due to the onset of a downstream leakage vortex. An increase in both the tip gap and exit Mach number resulted in an increase, followed by a decrease in the near-tip suction side heat transfer. On the near-tip pressure side, a slight increase in heat transfer was observed with increased tip gap and exit Mach number. In general, the suction side heat transfer is greater than the pressure side heat transfer as a result of the suction side leakage vortices. 


\section{Nomenclature}

C true chord of blade

$\mathrm{C}_{\mathrm{p}} \quad$ constant pressure specific heat

$\mathrm{h} \quad$ heat transfer coefficient

$\mathrm{k}_{\mathrm{a}} \quad$ thermal conductivity of air

$\mathrm{Ma} \quad$ Mach number

$\mathrm{Nu} \quad$ Nusselt number

$\mathrm{P} \quad$ pitch of blade

Pr Prandtl number

q" heat flux

Re Reynolds number

$\mathrm{S}$

$\mathrm{Sc}$ blade surface distance from stagnation point

Span of experimental blade

Schmidt number

Sh Sherwood number

St Stanton number

$\mathrm{T} \quad$ temperature

$\mathrm{Tu} \quad$ streamwise freestream turbulence intensity

U velocity

$\mathrm{x} \quad$ distance in axial direction

\section{Greek}

$\gamma \quad$ specific heat ratio of air

$\rho \quad$ local density of air

$\mu \quad$ dynamic viscosity of air

\section{Subscripts}

1 inlet conditions

2 exit conditions

aw adiabatic wall

gauge thin film gauge (surface) measurement

init, w initial wall

$\mathrm{L}$

local conditions

o stagnation

w wall

$\infty \quad$ mainstream 


\section{Introduction}

In order to allow for the rotation of the turbine blades in the hot section of a gas turbine engine, a small gap is allowed between the rotor blades and the stationary casing surrounding the turbine section. This gap unfortunately allows hot gases from the combustor exit to leak across the turbine blade tip. The pressure differential between the pressure and suction sides of the blade is the driving force for this tip leakage flow. Very high heat transfer levels are observed on the blade tip and near-tip region because of the thin boundary layer and the flow acceleration that characterizes tip leakage flow. An enhanced heat load on the blade tip leads to high thermal stress which normally results in the degradation of the blade tip material. As a result, a high cost of maintenance and parts replacement is associated with the turbine section of the engine.

An accurate understanding of the heat transfer characteristics on and around the blade tip, under representative engine conditions, will aid in developing effective cooling techniques that will help increase turbine blade life cycles. Tip leakage flow also serves to unload the turbine blade pressure side, thereby inducing unwanted aerodynamic losses. It is widely accepted that up to one third of the aerodynamic losses in the turbine section are caused by tip leakage flow.

\section{Summary of Past Literature}

Sjolander and Cao [1] studied the flow field in an idealized (simple blade geometry with stationary, linear cascade) turbine tip gap. They concluded that the flow separates from the pressure side tip corner and forms a well-organized chordwise vortex above the blade tip. The separation vortex induces a flow back toward the pressure corner, resulting in the formation of a secondary counter-rotating vortex. Thus, the unexpected region of attached flow near the pressure corner may have a reasonably high convective heat transfer coefficient, in addition to the high wall shear stress. Aerodynamic studies have been conducted to establish the benefit of using a squealer over the flat tip. Key and Arts [2] examined the flow characteristics of a flat and squealer turbine blade tip for different Mach number at a fixed tip gap value. Using a high-speed linear cascade, they concluded that the squealer tip provided a significant decrease in velocity through the tip gap, hence an overall reduction in aerodynamic losses when compared to the flat tip. Booth et al. [3] estimated that a tip clearance gap of $1 \%$ blade height could lead to losses of $1 \%$ - $3 \%$ in stage efficiency. 
The vast majority of turbomachinery literature has conducted their studies under stationary configurations. An early study on turbine blade heat transfer by Mayle and Metzger [4] concluded that the effects of relative motion of a blade to the shroud on heat transfer data are negligible. This conclusion was based on the argument that the pressure difference between the pressure and suction sides was the dominating factor in the tip-leakage flow phenomena. Yang et al. [5] have conducted a numerical study more recently to investigate the effect of rotation on the blade tip leakage flow and heat transfer. They used a flat tip and squealer tip geometry configuration from the $G E E^{3}$ first stage rotor blade. For the flat tip blade, Yang et al. stated that the relative motion of the endwall can reduce the leakage flow. They concluded that the relative motion of the endwall significantly increases the heat transfer on the flat blade tip.

Azad et al. [6] studied the flow and heat transfer on the first stage blade tip of an aircraft engine turbine $\left(\mathrm{GE}-\mathrm{E}^{3}\right)$. They presented the effects of tip gap clearance and freestream turbulence intensity level on the detailed heat-transfer coefficient distribution for both plain and squealer tips. Flow conditions in their cascade corresponded to an overall pressure ratio of 1.32 and an exit Reynolds number based on blade axial chord of $1.1 \times 10^{6}$. They used the transient liquid crystals technique and found that the overall heat-transfer coefficients on the squealer tip were lower than those of the plane tip. In addition, Azad et al. concluded that a larger tip clearance results in an increase in the overall heat transfer coefficients on the blade tip. They also determined that an increase in the turbulence intensity level from 6.1 to 9.7 percent increases the heat transfer coefficient by about 15-20 percent, along the leakage flow path.

Kwak and Han [7] used a hue detection-based transient liquid crystals technique to obtain heat transfer coefficient distribution on a blade tip, shroud and near-tip surfaces. The authors made their heat transfer measurements at tip clearances of 1.0, 1.5, and $2.5 \%$ of blade span. The Reynolds number based on exit velocity and blade axial chord was $1.1 \times 10^{6}$, and the overall pressure ratio was 1.2. They concluded that an increase in tip clearance increased heat transfer coefficient on the blade tip surface but the shroud and the blade suction side heat transfer increased and then decreased. In general, they determined that the heat transfer on the blade tip was higher than that on the shroud and on the near-tip suction and pressure sides. Interestingly, Kwak and Han did not observe any changes in the pressure side heat transfer coefficient distribution with increasing tip gap. 
Zhang et al. [8] studied, both experimentally and numerically, the heat transfer distribution on the tip of a high pressure turbine blade under transonic conditions $\left(\mathrm{M}_{\mathrm{exit}}=1.0\right.$,

$\operatorname{Re}_{\text {exit }}=1.27 \times 10^{6}$, gap $=1.5 \%$ chord). They experimentally obtained heat transfer results using the transient thermography technique while they used the CFD solver, HYDRA/PADRAM to numerically predict similar results. Zhang et al. concluded that the majority of the blade tip surface experiences supersonic flow which leads to reduced heat transfer. The authors observed oblique shock waves near the pressure side edge which were then reflected between the casing and the tip. Stripes were observed on the heat transfer distribution across the tip due to the multiple shock structure. Near the leading edge on the blade tip, the leakage flow remains subsonic resulting in higher heat transfer distribution.

Evident by the literature survey just outlined, several experimental and numerical studies have been done on turbine blade tip heat transfer in recent years. The majority of these studies in the open literature, however, have been carried out at low speed conditions and the region around the blade tip has not received significant attention. As such, adding to this body of work should provide more insights into the near-tip heat transfer on turbine blade surfaces. Testing at high speed, turbulent conditions also make the results representative of an actual engine.

\section{Experimental Setup and Instrumentation Wind Tunnel Facility}

The two-dimensional transonic cascade wind tunnel, shown in Figure 1.1, is a blow-down facility that is capable of sustaining a constant test section pressure for up to 25 seconds. Prior heat transfer research that has been performed in this facility includes work by Holmberg [9], Nix et al. [10], Smith et al. [11], Popp et al. [12], Carullo et al. [13], and Nasir et al. [14]. Air is supplied from high pressure air tanks that are charged up to $1380 \mathrm{kPa}$ (200 psig) prior to testing. A control valve regulates the flow from the air tanks to the test section. Cascade inlet pressures range from $20.7 \mathrm{kPa}$ (3 psig) to $69.0 \mathrm{kPa}$ (10 psig) depending on the objective test conditions. Between the control valve and the test section, the air passes through a passive heat exchanger, which heats the cascade inlet flow up to $115^{\circ} \mathrm{C}$. After the air passes through the heat exchanger, the air goes through a settling chamber and enters the test section before being exhausted to the atmosphere. Because of facility constraints, an increase in the exit Mach number is coupled to an 
increase in the exit Reynolds number. A square mesh turbulence grid of $48 \%$ solidity is used to generate the required turbulence intensity for the tests. The square mesh grid has bar widths of $1.91 \mathrm{~cm}$ (0.75 in.) and spaced to create $3.81 \times 3.81 \mathrm{~cm}^{2}\left(1.5 \times 1.5 \mathrm{in}^{2}\right)$ square openings. Hot-film anemometry measurements, performed by Nasir et al., show that the free-stream turbulence intensity level is 12 percent. This turbulence generating grid is placed at a distance 5.1C upstream from the test-blade leading edge. The corresponding normalized turbulence length scale was found to be 0.26 based on blade inlet pitch. The inlet Mach number is measured by a pitot static probe located 2.2C upstream of the center blade leading edge. The isentropic exit Mach number is decided based on the inlet total pressure (as measured by the pitot static probe) and the average wall static pressure measured $0.5 \mathrm{C}$ downstream of the cascade.

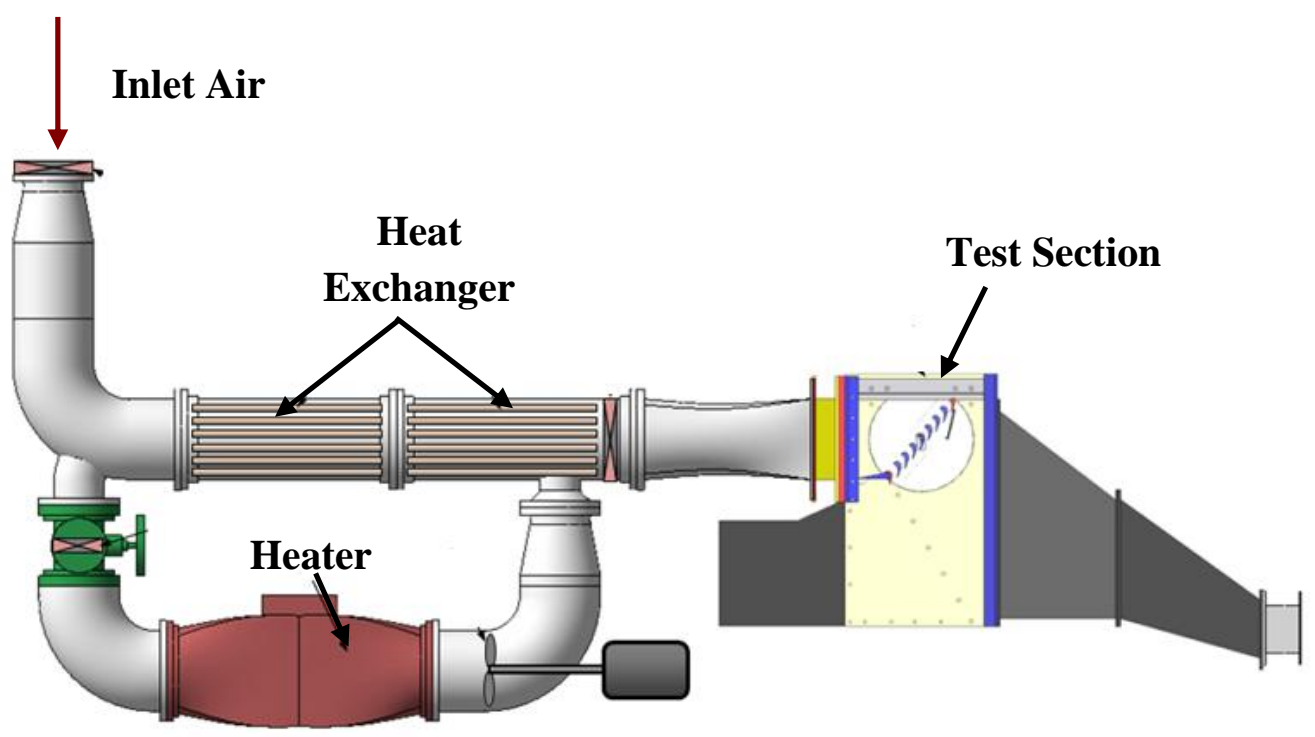

Figure 1.1 Transonic cascade wind tunnel

Table 1.1 Turbine blade geometry

\begin{tabular}{|c|c|}
\hline True chord & $69.9 \mathrm{~mm}(2.75 \mathrm{in})$. \\
\hline Axial chord & $58.2 \mathrm{~mm}(2.29$ in.) \\
\hline Pitch & $58.2 \mathrm{~mm}(2.29$ in. $)$ \\
\hline Span & $152.4 \mathrm{~mm}(6.00 \mathrm{in})$. \\
\hline Scaled engine blade span & $76.2 \mathrm{~mm}(3 \mathrm{in})$. \\
\hline Turning angle & $107.5^{\circ}$ \\
\hline
\end{tabular}


The turbine blade tested in these experiments is similar in geometry to a first stage turbine blade. The blade was scaled two times so that the nominal exit Reynolds number utilized in these tests would be more easily attained, in addition to the need to make the blades fit in the test section with minimal facility alterations. Table 1.1 summarizes the geometry of the turbine blade.

A diagram of the blade cascade, showing also the infrared (IR) window for heat transfer measurements, is provided in Figure 1.2. Determined by the blade geometry and the test section size, the cascade consists of seven full blades and two partial blades (not shown), which results in eight full passages. A tailboard placed at the blade exit angle aids in creating periodic flow through the cascade. The central blade is fully instrumented for heat transfer measurements. All three central blades in the cascade are adjusted to obtain the desired tip gap in the experiments. The remaining outer blades are fixed to the endwall on both ends, thereby maintaining no tip gaps.

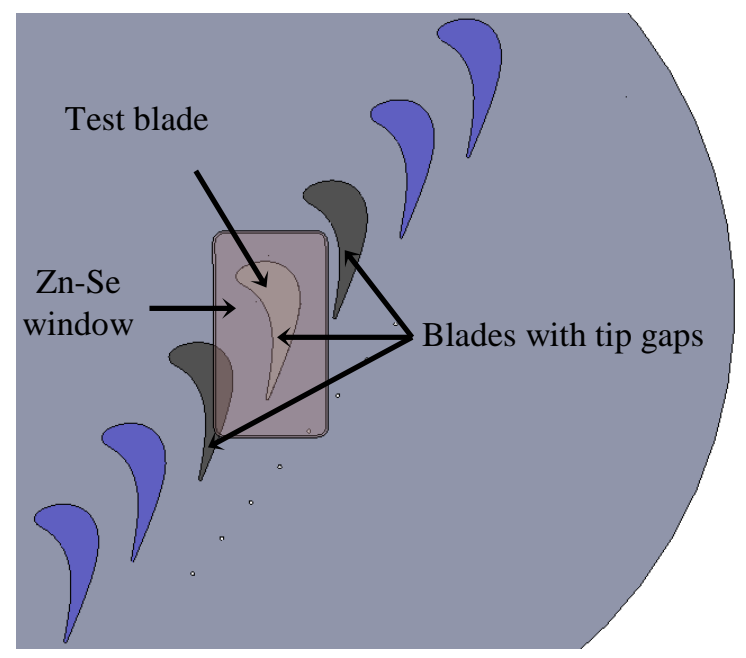

Figure 1.2 Cascade diagram showing the blades

Figure 1.3 shows how the blade tip gap is defined. Two tip gaps of $0.6985 \mathrm{~mm}(0.0275$ in.) and $1.397 \mathrm{~mm}(0.055 \mathrm{in}$.) corresponding to $0.9 \%$ and $1.8 \%$ of engine blade span were used in the present study. 


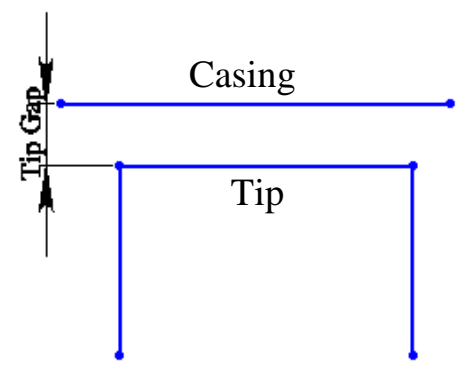

\section{Figure 1.3 Definition of tip clearance}

\section{Heat Transfer Measurements IR Technique}

All the internal surfaces of the cascade, including the three central blade tips were painted with a thin flat black paint coat in order to increase the emissivity of the surfaces and reduce the reflected radiation from the metallic surfaces. The FLIR A325 infrared (IR) camera was used to obtain heat transfer measurements on the central blade tip through a Zinc Selenide ( $\mathrm{ZnSe}$ ) window placed in-between the IR camera and the center test blade (see Figure 1.2). The IR camera produces spatially resolved surface temperature data at $15 \mathrm{~Hz}$ frequency at $320 \times 240$ pixels image resolution. An Aluminum endwall contains a 4.5 in. $\times 2.5$ in. view slot which accommodates the $\mathrm{ZnSe}$ window for the IR camera. The $\mathrm{ZnSe}$ window has a transmissivity of 0.97 in order to minimize the absorption and reflection of radiation coming to the IR camera. The IR camera was calibrated at the start of a set of experiments, using an in situ thermocouple placed on the blade tip. The Macor material used to manufacture the instrumented portion of the test blade has a very low thermal conductivity. The temperature measurement window for the experimental runs is a short time period of about 5 seconds. This results into a penetration depth $\sqrt{ }$ at of about $1.9 \mathrm{~mm}(0.08 \mathrm{in}$.) for the data acquisition time period. Heat transfer coefficients are then obtained using the solution to the one-dimensional semi-infinite transient conduction equation with convective boundary condition. This technique has extensively been used and documented by Ekkad et al. [15].

$$
\frac{T_{w}-T_{i n i t, w}}{T_{\infty}-T_{i n i t, w}}=1-\left[\exp \left(\frac{h^{2} \alpha t}{k^{2}}\right)\right]\left[\operatorname{erfc}\left(\frac{h \sqrt{\alpha t}}{k}\right)\right]
$$


Equation 1.1 is solved for every pixel location on the blade tip as a function of time since all the terms in the equation are known except the heat transfer coefficient.

The solution to Equation 1.1 assumes that the heat transfer coefficient is constant throughout the entire time of the data reduction. This is not necessarily the case in this experiment due to the unsteadiness caused by the startup effects in the wind tunnel. In order to eliminate that assumption, the heat flux distribution in time, at each pixel location, could be obtained from which the heat transfer coefficient and adiabatic wall temperature may be calculated.

\section{Thin Film Gages}

Heat transfer measurements on the near-tip pressure and suction sides were made with thin film gauges that allow for high frequency measurements on the blade surface with minimal flow disruption. Thin film gauges were originally developed by Schultz and Jones [16] and variations of the original design have been used by Doorly and Oldfield [17] and Dunn [18], to name a few. The thin film gauges that were used in these experiments are two-layer thin film gauges similar to the gauges developed by Doorly and Oldfield [17].

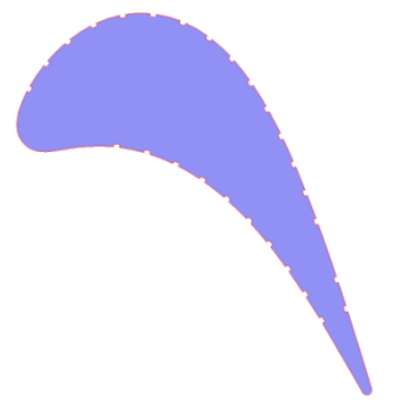

Figure 1.4 Blade profile showing thin film gage locations

Figure 1.4 shows the gage locations, chord-wise, around the blade pressure and suction sides. Unfortunately, few gages were lost during the runs and these gages are eliminated from the Figure. Each thin film gauge consists of a platinum sensor that is $2.54 \mathrm{~mm}(0.1 \mathrm{in}$.) wide that attach copper leads which are sputtered to a Upilex $(k=0.289 \mathrm{~W} / \mathrm{mK})$ sheet that is $50 \mu \mathrm{m}$ thick. The Upilex sheet with the gauges is attached to the Macor (low thermal conductivity of $k=1.46$ $\mathrm{W} / \mathrm{mK}$ ) portion of the central blade. A photograph of the gauges installed on the blade is shown in Figure 1.5. The platinum sensor was placed at $94 \%$ of engine blade span $(4.57 \mathrm{~mm}$ from the tip) and a total of 32 thin film gauges were instrumented on the blade. 


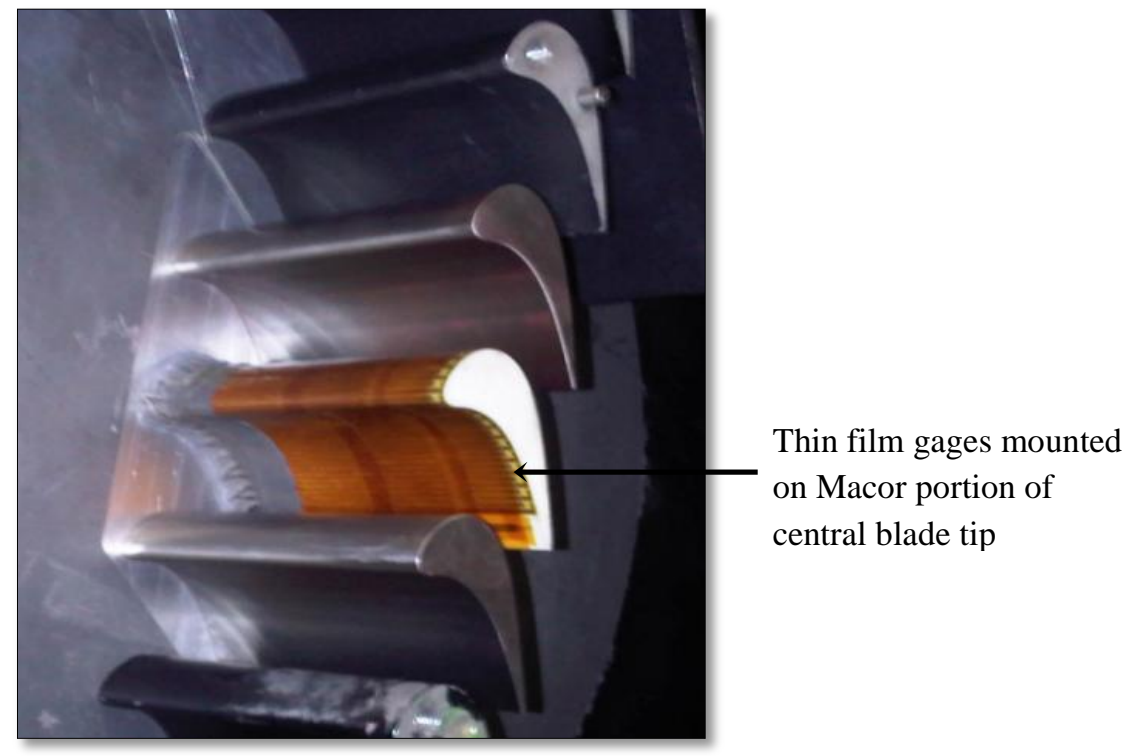

Figure 1.5 Thin film gages in wind tunnel test section

Thin film gauges are used to measure a change in temperature on the surface of the blade. The platinum sensor of the thin film gauge changes resistance with temperature and each gauge is calibrated prior to testing. Since the gauge changes resistance with temperature, the gauge is used as one arm of a Wheatstone bridge circuit. The Wheatstone bridge used in these experiments is described by Joe [19]. The change in voltage across the bridge during the experiment is sampled at $1 \mathrm{kHz}$ during the experiment using a 16 Bit NI SCXI-1600 data acquisition system. The data from up to 32 gauges can be recorded in this facility during a single test.

To reduce the heat transfer data, several steps must be taken. The voltage output from each Wheatstone bridge is converted into surface temperature using the gauge calibration and basic Wheatstone bridge operating principles. Next, the heat flux for each gauge is calculated by using a finite-difference code developed by Cress [20]. The finite-difference code uses the time history of the surface temperature of a gauge as a boundary condition and solves the onedimensional, transient heat conduction equation. Over the majority of the blade, the conduction is assumed to be semi-infinite since the Macor conducts heat very slowly. Once the heat flux for each gage is determined, the corresponding heat transfer coefficient can then be calculated by using,

$$
q^{\prime \prime}=h\left(T_{a w}-T_{w}\right)
$$


Where $T_{a w}$ is the adiabatic wall temperature, $T_{w}$ is the blade surface temperature measured using the platinum thin film gauge and $h$ is the heat transfer coefficient. Equation 1.2 can be rewritten as:

$$
q^{\prime \prime}=h\left(T_{\infty}-T_{w}\right)-h\left(T_{\infty}-T_{a w}\right)
$$

Using Equation 1.3, we can determine $h$ and $T_{a w}$ by plotting $q$ " versus $\left(T_{\infty}-T_{w}\right)$. The result gives us a line of the form $y=m x+b$, where the slope of the line is the heat transfer coefficient and the $\mathrm{x}$-intercept of the line is $\left(T_{\infty}-T_{a w}\right)$. The slope is determined using a least squares linear regression fit of the plotted data. More explanation of this technique can be seen in Smith et al. [11] and Popp et al. [12]. The heat transfer coefficient can then be nondimensionalized by calculating the Nusselt number using,

$$
N u=\frac{h \cdot C}{k}
$$

The heat transfer coefficient can also be nondimensionalized in terms of the Stanton number given by,

$$
S t=\frac{h}{\rho \cdot U \cdot C_{p}}
$$

The inlet endwall momentum and thermal boundary layers were measured at $0.18 \mathrm{C}$ upstream of the test blade leading edge. A kiel probe projected into the flow, at different distances from the endwall, was used to measure the total pressure needed to obtain the flow velocity profile in the boundary layer. Similarly, at the same upstream location, a K-type thermocouple was used afterwards to measure the temperature profile in the boundary layer. The pressure and temperature data are used to obtain the respective nondimensionalized velocity and temperature profiles within the endwall boundary layers. Plots of the resulting momentum and thermal boundary layers as a function of nondimensionalized perpendicular distance into the fluid, within the boundary layer, are shown in Figure 1.6.

In general, thinner (compared to the tip gap) inlet endwall momentum and thermal boundary layer thicknesses are desirable. A smaller momentum boundary layer ensures that secondary flows in the tip gap are reduced so that the three dimensional flow development would be dominated by the tip leakage flow. The relatively thick thermal boundary layer obtained could 
affect the heat transfer levels obtained in this study since the upstream temperature is used as the reference temperature for data post processing. Figure 1.7 shows a typical adiabatic wall and blade surface temperature time histories during a run.

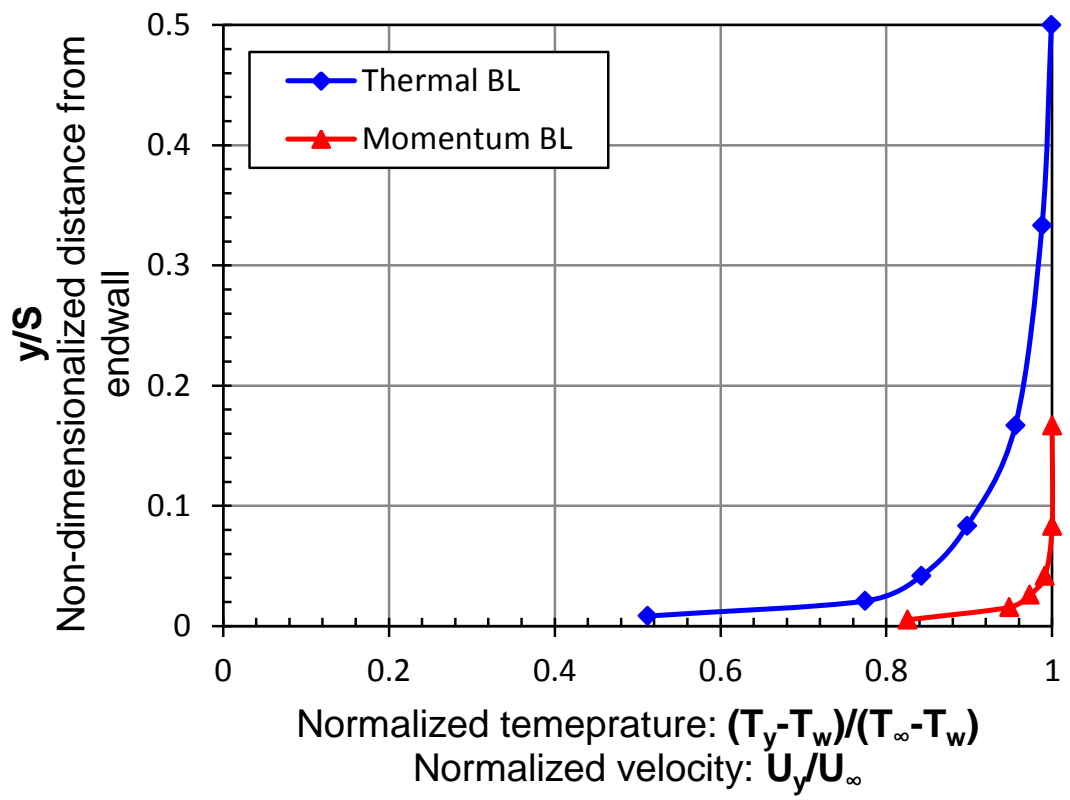

Figure 1.6 Inlet endwall boundary layer profile measured approximately $0.18 \mathrm{C}$ upstream of the test blade leading edge

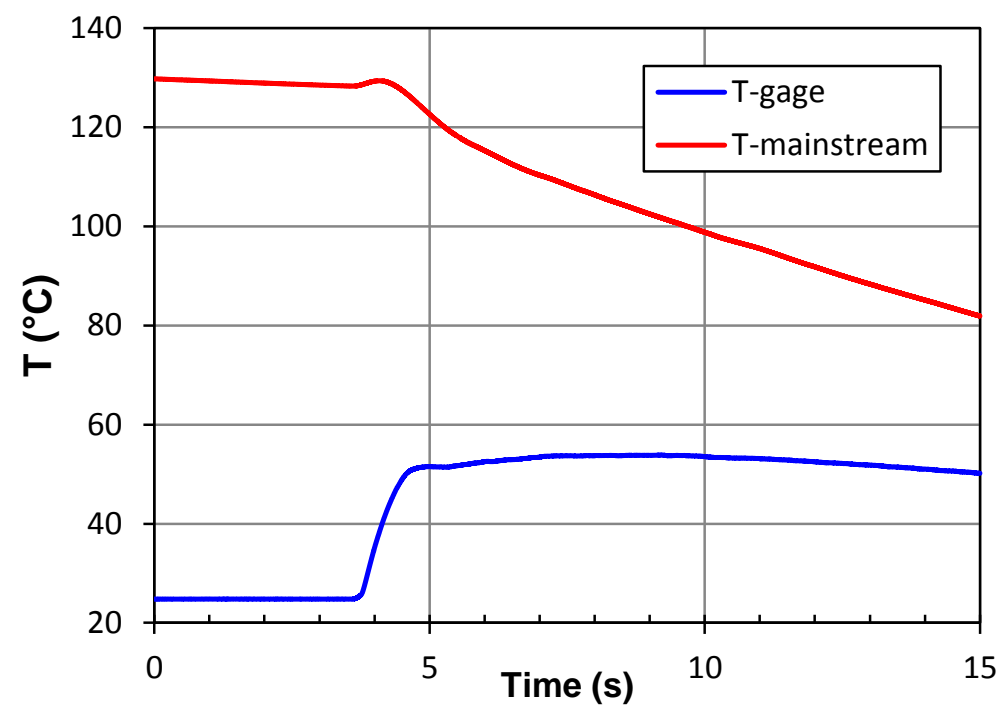

Figure 1.7 Typical mainstream and blade surface temperatures history 


\section{Uncertainty Analysis}

The experimental uncertainty of the heat transfer measurements was calculated using the procedure developed by Moffat [21]. The analysis took into account the bias error and precision error. For the near-tip measurements, an uncertainty was calculated for each gauge at the design condition. The total uncertainty of the heat transfer coefficient for the gauges ranged between $8.5 \%$ and $11.5 \%$ with the bias error contributing the majority to the total uncertainty. For the blade tip heat transfer measurements using the IR technique, the average uncertainty in the heat transfer coefficient values was found to be $\pm 9.5 \%$. The uncertainty in the heat transfer data around the edges of the blade tip is expected to be higher than the values reported. The breakdown in the 1-D assumption near the edges is the reason for the higher uncertainty since the heat transfer coefficients are obtained based on the premise that the assumption holds true. In estimating the uncertainty for both measurement techniques, the single sample experiment procedure for determining uncertainties was used.

\section{Blade Static Pressure Distribution at Midspan}

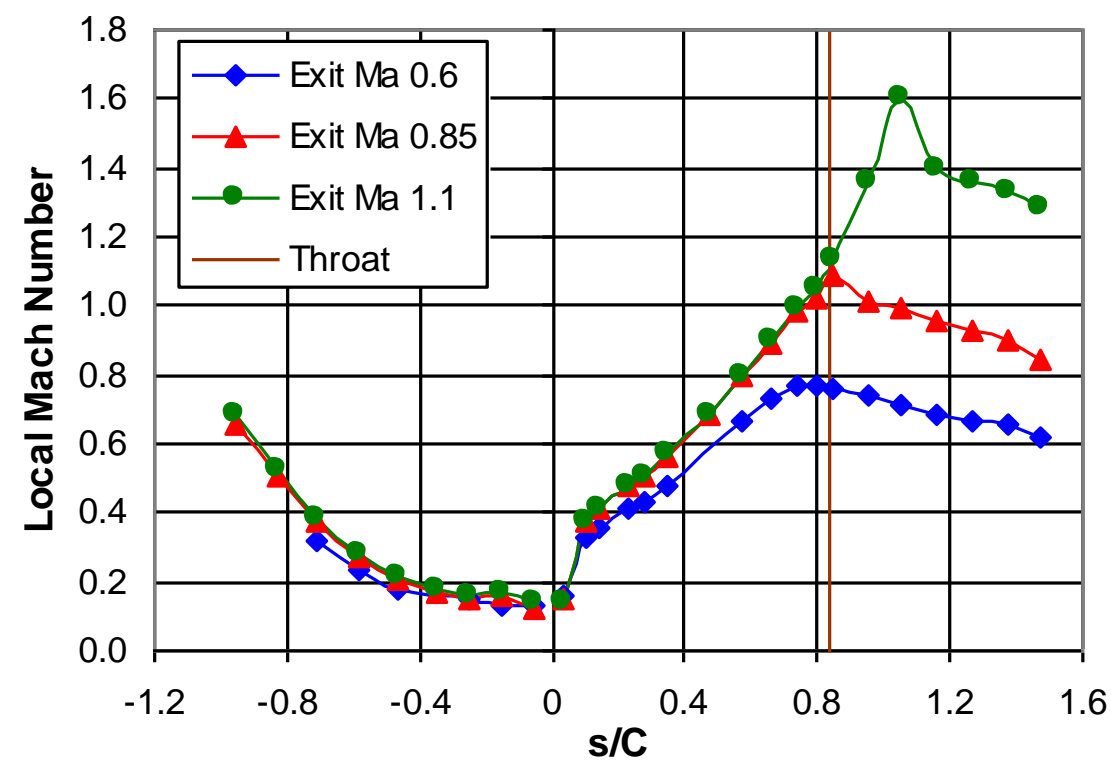

Figure 1.8 Local Mach number distribution over blade

Carullo et al. [13] measured the local Mach number distribution on a geometrically identical blade to that of the present study at the blade midspan. There was no tip clearance gap 
associated with their experiment. Since the tip leakage flow is driven by the pressure difference between the pressure and suction sides, the local Mach number distribution at blade midspan is expected to shed some light on the subsequent heat transfer data analysis at the tip. Figure 1.8 shows the local Mach number distributions, obtained by Carullo et al., on the blade surface for three exit Mach number conditions at blade midspan. For all three Mach number cases, a steady flow velocity increase is seen on the suction side up to $\mathrm{s} / \mathrm{C}=0.84$. Beyond this area, the blade geometric throat, the flow velocity drops for the exit Mach 0.6 and 0.85 cases. The exit Mach 1.1 case however showed continued flow velocity increase before an eventual deceleration. On the blade pressure side, the flow velocity increases for the most part representing an overall pressure surface flow acceleration trend.

\section{Test Conditions}

Heat transfer measurements were performed at exit Mach numbers of 0.7, 0.85, and 1.05. The tip clearance gaps were varied between $0.9 \%$ and $1.8 \%$ of engine blade span at each exit Mach number. The test matrix resulted in heat transfer data for six different flow conditions for the near-tip heat transfer. Heat transfer measurements, for each measurement technique, were performed at least two times for one test matrix to establish repeatability. Table 1.2 provides the flow and heat transfer conditions for each test. The exit Reynolds number coupled with the exit Mach number is based on engine blade span and is defined by,

$$
\operatorname{Re}_{2}=\frac{\rho_{2} \cdot U_{2} \cdot H}{\mu_{2}}
$$

where the average pressure and temperature data during the test run were used to calculate the exit Reynolds number.

Table 1.2 Test conditions for each case

\begin{tabular}{|c|c|c|}
\hline & Exit Ma & Tip Gap (\%) \\
\hline Case 1 & 0.74 & 0.9 \\
\hline Case 2 & 0.70 & 1.8 \\
\hline Case 3 & 0.85 & 0.9 \\
\hline Case 4 & 0.85 & 1.8 \\
\hline Case 5 & 1.04 & 0.9 \\
\hline Case 6 & 1.05 & 1.8 \\
\hline
\end{tabular}


Only four flow conditions (cases 3-6) were obtained for the blade tip heat transfer. Also, case 3 is the design condition for the tests at Mach 0.85 with $0.9 \%$ tip gap.

\section{Flow Visualization}

\section{Blade Tip}

Figure 1.9 shows the flow visualization results on the blade tip at exit Mach 0.8 at $0.9 \%$ tip gap (Figure 1.9a) and 1.8\% tip gap (Figure 1.9b).

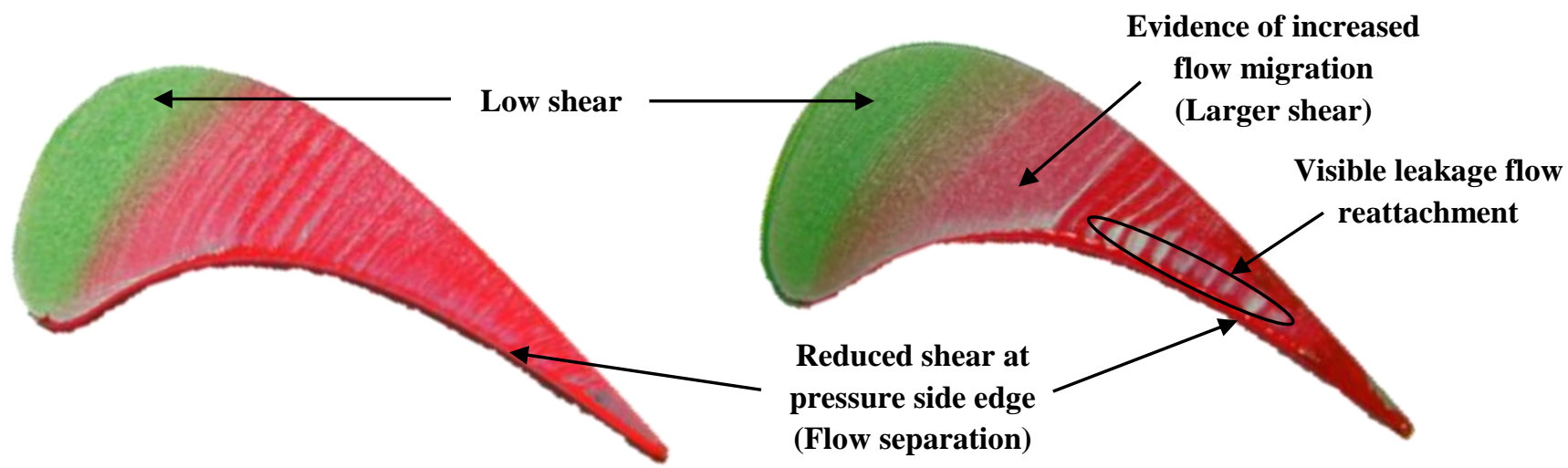
(a) $0.9 \%$ Tip gap
(b) $1.8 \%$ Tip gap

Figure 1.9 Flow visualization on the blade tip at exit Mach 0.85

Figures 1.9a and 1.9b reveal that a small shear is observed around the leading edge of the blade tip. This is clear because the blade tip pigment, applied before the run, still remains relatively uncontaminated. The pressure side leakage flow is not seen to have been carried across the blade tip in this region for both cases. Further downstream, a stronger and more visible pressure side leakage flow is carried across the tip surface, as evident by the pressure side pigment smeared on the tip. This leakage flow however, seems to impose a relatively significant shear effect (flow migration) in the $1.8 \%$ tip gap case. Such an observation is consistent with the fact that a larger tip gap implies that more leakage flow will enter the tip clearance gap. Around the pressure side edge, especially for the $1.8 \%$ tip gap case, areas of significant shear can be seen. These areas that are exposed to larger shear trace the reattachment line of the tip leakage 
flow on the blade tip surface. Much closer to the pressure side edge, lower shear is observed confirming that flow separation occurred in that vicinity.

\section{Suction Side}

Figure 1.10a shows the suction side visualization for $0.9 \%$ tip clearance case. The most visible feature is the tip leakage vortex that forms as a result of the interaction between the tip leakage flow and the passage flow near the blade suction side. At about s/C $\sim 0.7$ from the leading edge on the suction side, a line separating the flow going towards the blade mid-span and the flow towards the tip forms in the streamwise direction. As was pointed by Key and Arts [2], this line is called the impingement line. Further downstream along the blade, the size of the tip leakage vortex increases, and it affects a larger spanwise extent of the blade.

At about 0.13 inch ( $4.3 \%$ of engine blade span) from the tip, the suction side streamlines that head towards the tip appear to meet up with the flow that came from the pressure side and blade tip. The line where pigments from these three surfaces (pressure side, suction side \& blade tip) meet is considered a liftoff line.

In comparison to the $0.9 \%$ tip clearance case, the impingement line for the $1.8 \%$ tip gap seems to have disappeared as shown in Figure 1.10b. Towards the trailing edge, the leakage vortex appears comparatively weaker which might be due to a reduced velocity of the tip leakage flow compared to that with the smaller tip gap.

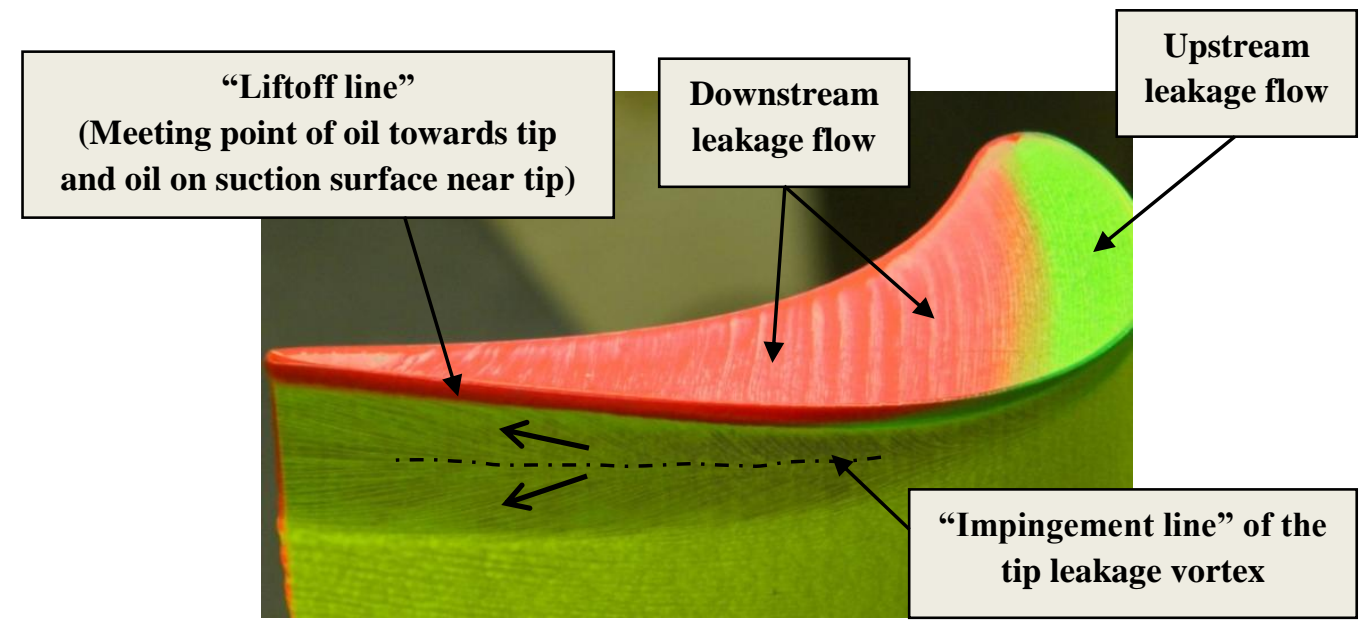

(a) 


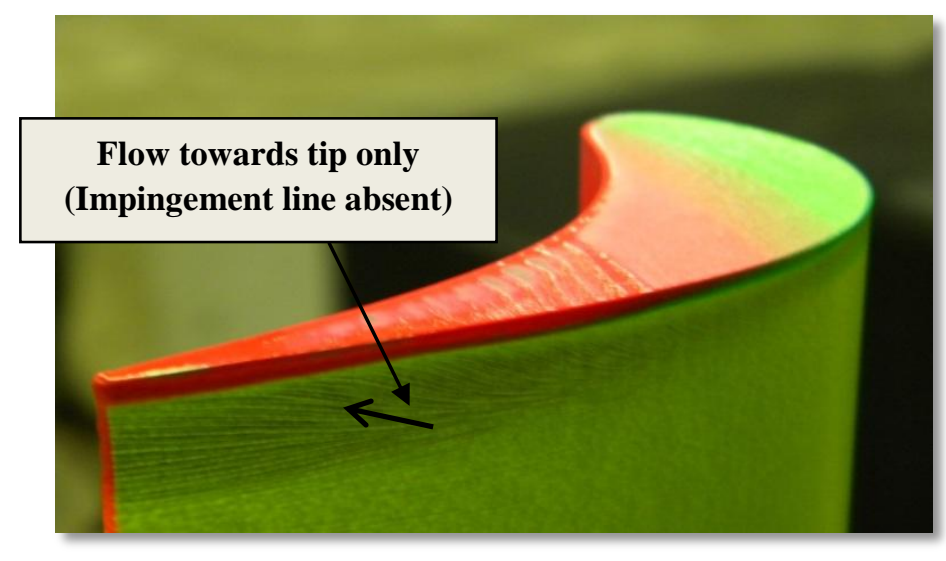

(b)

Figure 1.10 Suction side flow visualization at $M=0.85$ with a tip clearance of (a) $0.9 \%$ (baseline) and (b) $1.8 \%$

\section{Blade Heat Transfer Distribution}

\section{Heat Transfer on the Blade Tip}

Figures 1.11 and 1.12 present the detailed heat transfer coefficients for the blade tip at exit Mach 0.85 and 1.05 respectively with $0.9 \%$ and $1.8 \%$ tip gaps. High heat transfer is observed at the leading edge locations where hot air strikes the blade surface head-on, at the tip and near-tip regions, before it gets deflected as it progresses within the tip gap. Immediately after the leading edge, a large region of low heat transfer is seen in both cases from each figure. Supported by the flow visualization in Figure 1.9, the 'shear' in this region is relatively low due to the lower pressure and suction sides pressure differential. This so-called "sweet spot" has been observed by Bunker et al. [22], whose study was conducted at flow conditions of exit Mach 0.75 with varying turbulent intensities of five and nine percent. In the present study, the "sweet spot" region is larger for the lower tip gap cases of $0.9 \%$. As previously mentioned, an in situ thermocouple was placed on the tip for IR camera calibration. The disturbance in heat transfer coefficient caused by this thermocouple is marked in Figure 1.11b. 


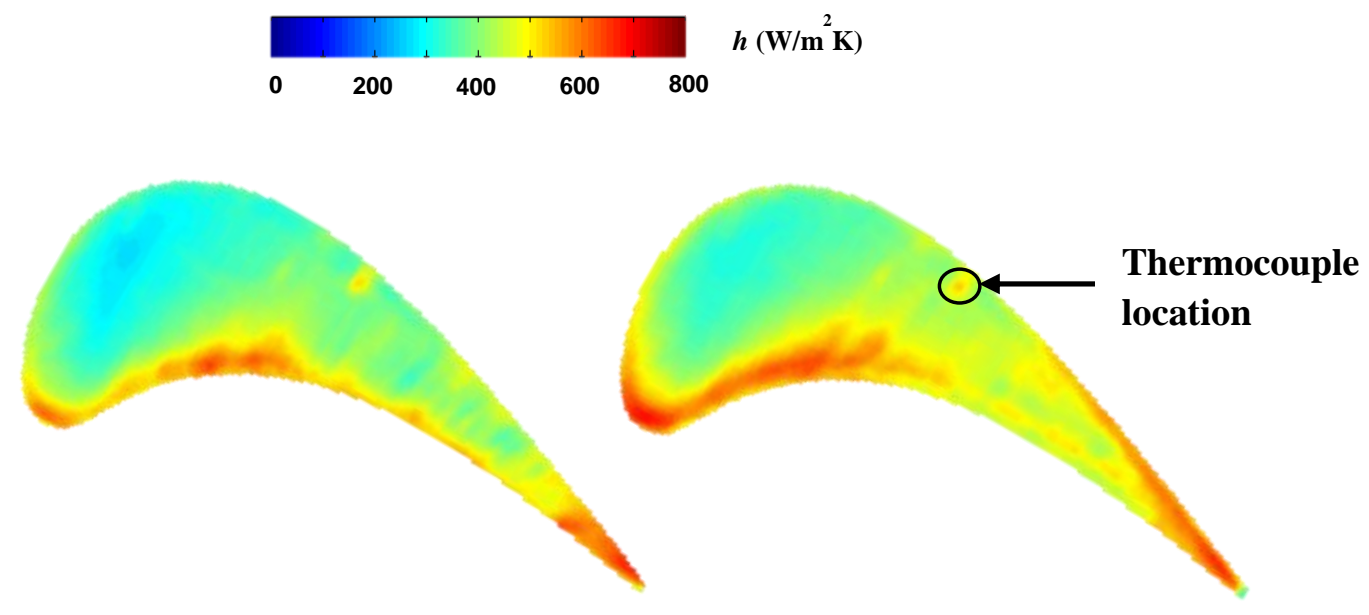

(a) $0.9 \%$ Tip gap

(b) $1.8 \%$ Tip gap

Figure 1.11 Heat transfer coefficient distribution on the blade tip at exit Mach 0.85
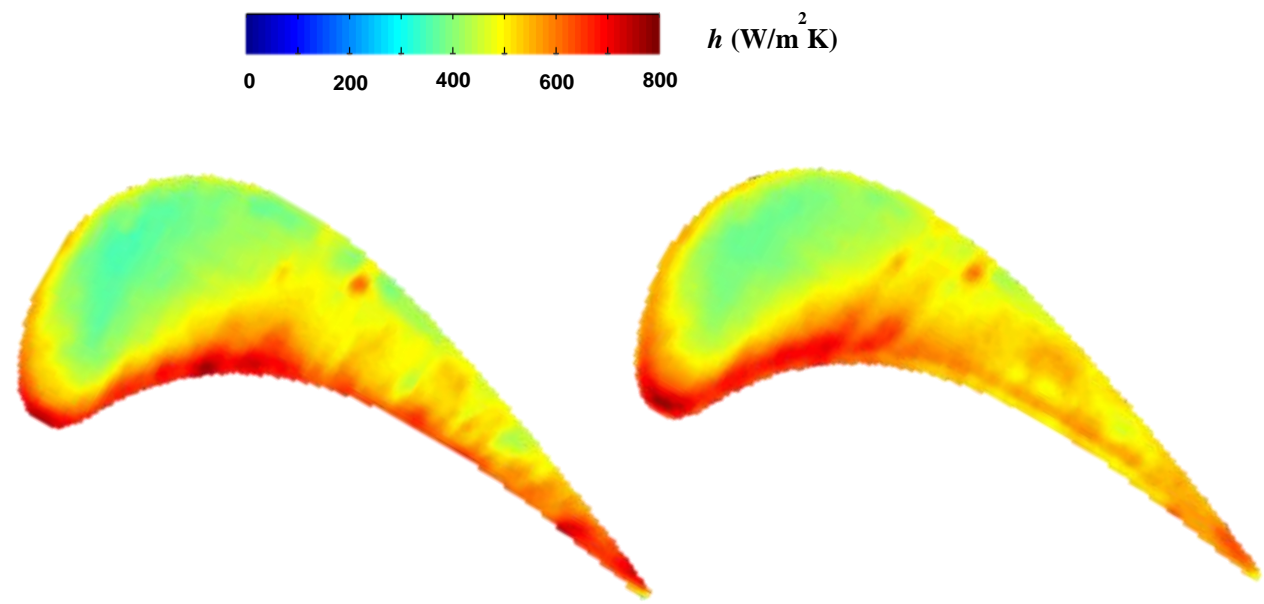

$\begin{array}{ll}\text { (a) } 0.9 \% \text { Tip gap } & \text { (b) } 1.8 \% \text { Tip gap }\end{array}$

Figure 1.12 Heat transfer coefficient distribution on the blade tip at exit Mach 1.05

Kwak et al. [7], in a similar study at exit Mach 0.59, observed high heat transfer coefficients near the pressure side between $20 \sim 70 \%$ of blade axial chord. They maintain that this region corresponds to the maximum leakage flow region caused by the large pressure difference between the blade pressure and suction sides. The present study, Figures 1.11 and 1.12, also shows high heat transfer coefficients near the pressure side edge. As flow enters the clearance gap, flow separation occurs at the pressure side tip edge as evident by the low heat transfer in that region. A visible indication of flow separation near the pressure side edge of the 
blade tip is shown in the flow visualization on Figure 1.9b. Separation bubbles may have been formed within the confines of the flow separation areas which were also observed by Sjolander and Cao [1]. Subsequent reattachment occurs on the tip surface towards the suction side. As the tip gap increases, the separation region near the pressure side edge also increases as shown in Figures 1.11 and 1.12. This observation was also made by Nasir et al. [23] and Kwak et al.[7], although both tests were conducted at lower speed conditions.

The range of heat transfer coefficient values is from about $270-800 \mathrm{~W} / \mathrm{m}^{2} \mathrm{~K}$ for all the results. Overall, the high heat transfer area is larger for the 1.8\% tip gap cases as expected. A larger tip gap allows more leakage flow from the pressure side to the suction side thus an enhanced heat load is noticed. A comparison between Figures 1.11a and 1.12a indicates the effect of an increase in the exit Mach number on heat transfer. An increase in exit Mach number results in an overall increase in the blade tip heat transfer coefficients. In a similar study by Zhang et al. on blade tip heat transfer at $\mathrm{M}_{\text {exit }}=1.0$, the authors reported higher heat transfer distribution throughout the leading edge region. This result does not exactly compare with the exit Mach 1.05 case in the present study where a low heat transfer region exists near the leading edge suction side region. This difference might be due to the dissimilarity of the blade geometry used in in both experiments. The separation and reattachment region, near the pressure side edge, were seen in the heat transfer distribution in both cases however.

\section{Averaged Heat Transfer Coefficient}

Figures 1.13 and 1.14 detail the circumferential averaged heat transfer coefficients on the blade tip at a fixed exit Mach number of 0.85, and for a given tip clearance of $0.9 \%$ respectively. The local heat transfer coefficients are averaged at a given $\mathrm{x} / \mathrm{C}_{\mathrm{x}}$ location. It can be seen from Figure 1.13 that an increase in the tip clearance gap results in an overall increase in the heat transfer coefficients. Specifically, for a fixed exit Mach number of 0.85, the averaged blade tip heat transfer increased by $12 \%$ for an increased tip clearance gap from $0.9 \%$ to $1.8 \%$ based on engine blade span. In the same vein, an increase in the Mach number shows an increase in heat transfer. A $24 \%$ increase in heat transfer was obtained at $0.9 \%$ tip clearance for an exit Mach number increase from 0.85 to 1.05 . It is noteworthy to mention that there is higher uncertainty in 
heat transfer coefficient at the trailing edge due to 2-D heat transfer and relative breakdown in semi-infinite assumption.

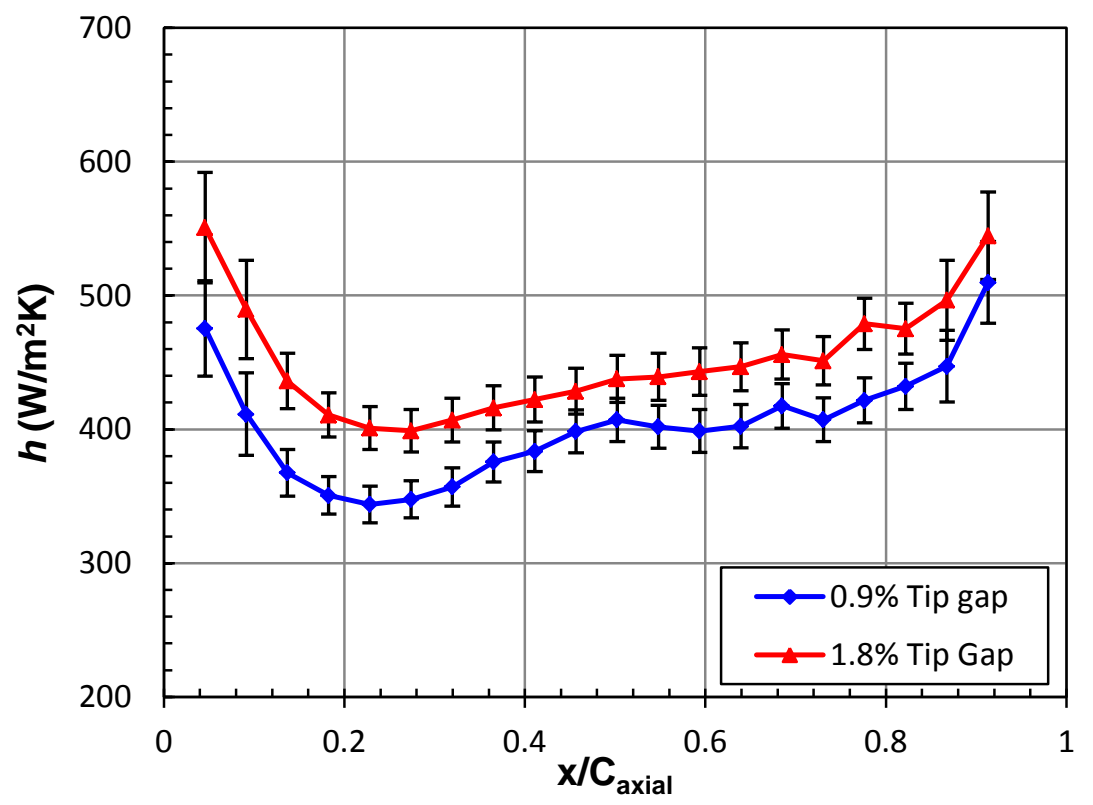

Figure 1.13 Circumferential averaged heat transfer on the blade tip at exit Mach 0.85

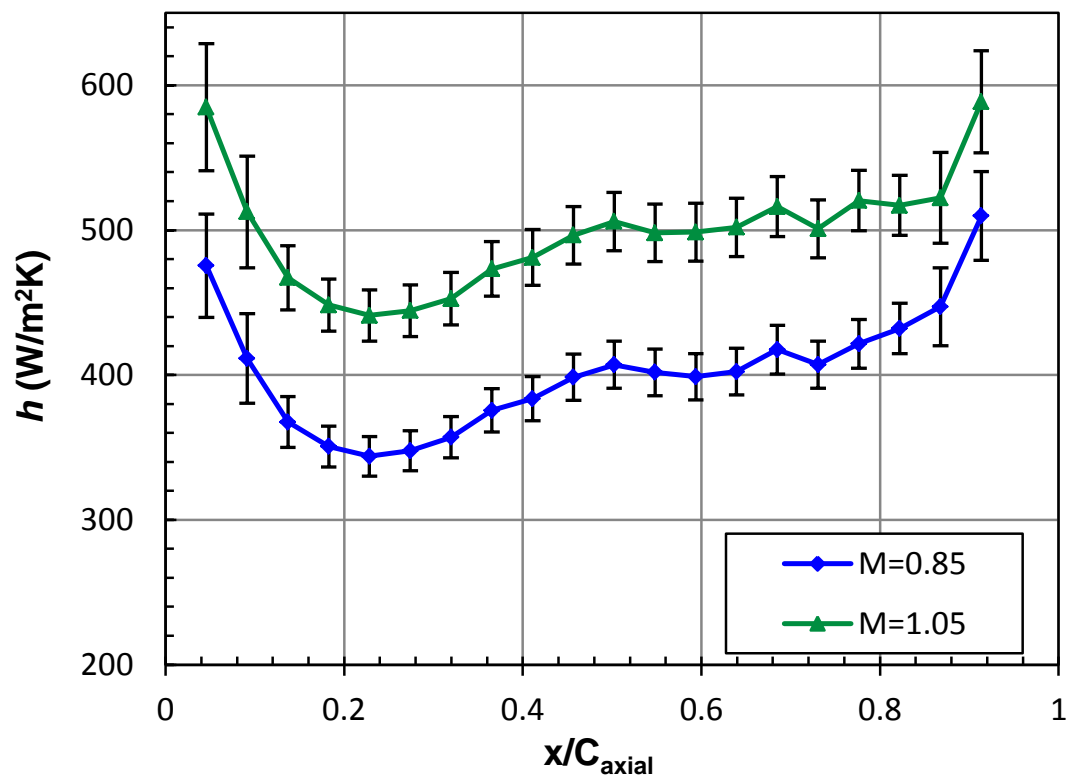

Figure 1.14 Circumferential averaged heat transfer on the blade tip for $0.9 \%$ tip gap 
Heat Transfer on the Near-Tip Region

\section{Basic Heat Transfer Characteristics at the Design Condition}

In the near-tip region at the design condition, the leakage flow from the gap has a dominant effect on the local heat transfer. Figure 1.15 shows the Nusselt number distribution along the blade near tip region.

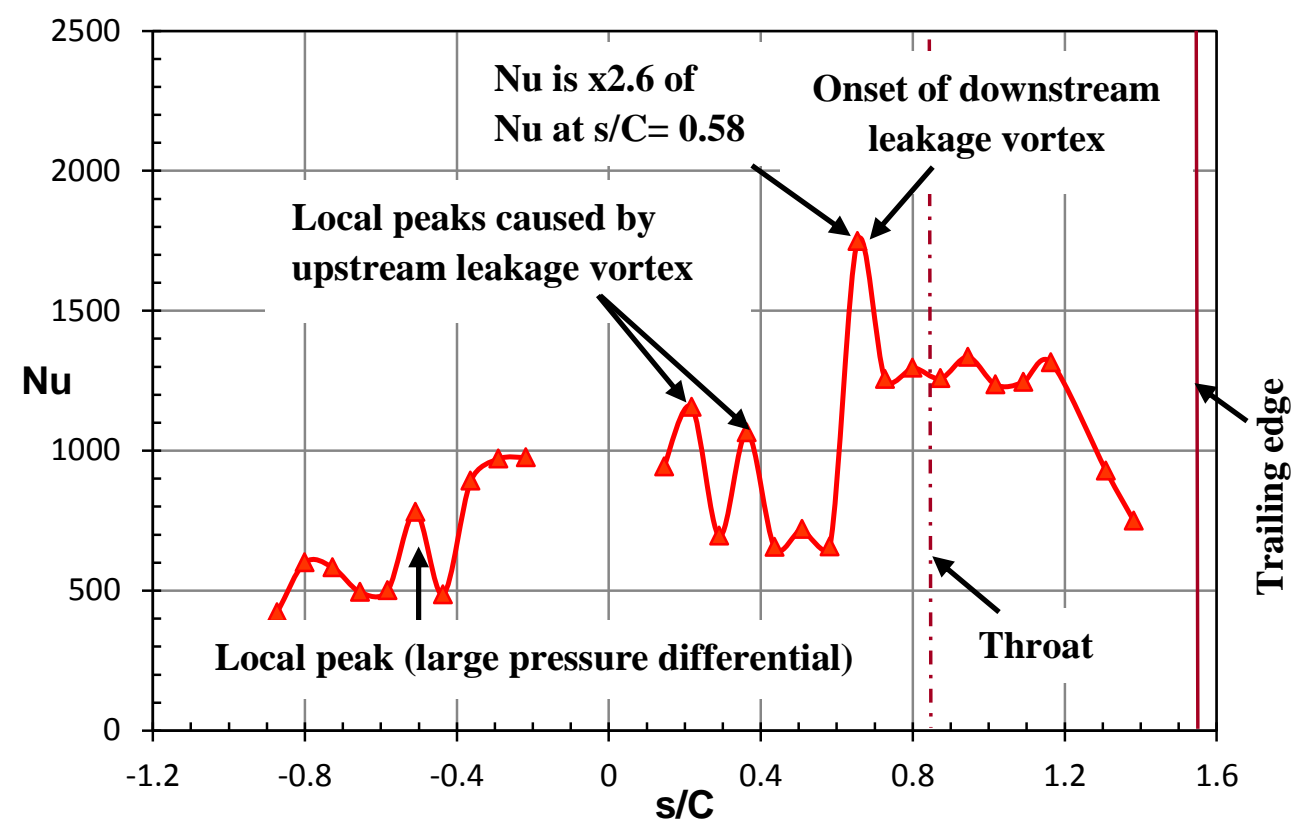

Figure 1.15 Heat transfer distribution at the design condition with exit Mach 0.85, 0.9\% tip gap

There are three local peaks in Nusselt numbers near the tip on the suction side surface originating from two categories of leakage flow. The flow visualization in Figure 1.10 clearly shows the two distinct regions that are dominated by these leakage flows across the blade tip. This partitioning is because the leakage flow into the tip gap can be divided into the upstream and the downstream tip leakage flow regions. The upstream leakage vortex originates from the leakage flow that occurred towards the blade leading. This upstream leakage flow is fairly weak due to the relatively low pressure differential that drives the flow from the pressure side to the suction side. The downstream leakage vortex, on the other hand, originates from the pressure side leakage flow that gets carried across the tip to the suction side. Each of these flows 
develops into two leakage vortices. Detailed tip leakage behavior is provided by Moore and Tilton [24].

In the upstream region, two small region/peaks of high heat transfer are generated due to the leakage flow from the upstream portion of the gap, as shown in Figure 1.15. The local peak between $0.29 \leq \mathrm{s} / \mathrm{C} \leq 0.36$ represents a $53 \%$ spike in Nusselt number. As expected, this upstream region is confined to $\mathrm{s} / \mathrm{C} \leq 0.44$. The reason is because the upstream leakage flow, affecting the surface heat transfer, separates from the blade surface when it encounters the flow in the passage on the blade suction side. Then, a new high heat transfer region, due to the downstream leakage vortex, emerges after a clear distinction between these two regions (evident by the locally minimum value at $\mathrm{s} / \mathrm{C}=0.58$ ). This leakage vortex impinges on the suction side surface, creating a relatively high heat transfer region along the leakage vortex path. The peak value due to this leakage vortex is located at $\mathrm{s} / \mathrm{C}=0.66$ and it represents a Nusselt number increase of $2 \frac{1}{2}$ times relative to that obtained at an immediate s/C location of 0.58 . The Nusselt number immediately drops after this peak value because a sizeable portion of the strong downstream vortex separates from the blade surface. The effect of the downstream leakage vortex is nonetheless sustained up to an s/C location of 1.2. As s/C further increases, the level of Nusselt number in the near-tip region drops. This confirms that the leakage vortex became larger but weakened as indicated by the flow visualization.

Results on the pressure side, also from Figure 1.15, show high heat transfer towards the leading edge with a sharp decline away from the leading edge. A local peak in heat transfer around mid-chord, corresponding to a $60 \%$ rise based on the preceding chord location, on the pressure side is noted. Blade pressure distribution on the pressure side obtained by Key and Arts [2] could aid in understanding the flow, and hence the heat transfer result at this near-mid-chord pressure side region. Their results were obtained near the tip at 0.9 exit Mach number, $97.3 \%$ span, and with a $1.34 \%$ of blade height tip gap. The authors observed that the region around the mid-chord presented the largest pressure differential between the pressure and suction sides. They concluded that this is the portion where the highest velocities through the tip gap are expected. This enhanced mass flow around the mid-chord section, in the present study, could be the reason for the local peak in heat transfer on the pressure side as a larger mass of air is pulled across the tip. Beyond this peak, lower heat transfer coefficients are observed in an increasing then decreasing trend. 


\section{Comparison with Rhee and Cho [25]}

Figure 1.16 represents the Nusselt number near the blade tip in the present study compared to the near tip heat transfer study by Rhee and Cho [25] at low speed. It is noted that the blade geometry, cascade arrangement, and flow conditions of their study are different from that of this present study. Their study was conducted in an annular turbine cascade with sixteen blades, a $2.5 \%$ of blade chord tip clearance, an exit Reynolds number of $1.8 \times 10^{5}$ based on blade true chord and a 3\% inlet turbulence intensity. The naphthalene sublimation technique was used to obtain time-averaged mass transfer coefficients. In order to make a comparison with the present study, the heat-mass transfer analogy, Equation 1.7, was used to acquire heat transfer results from the Sherwood number distribution provided by Rhee and Cho.

$$
N u=\left(\left(\operatorname{Pr} / S_{C}\right)^{\frac{1}{3}}\right) S h
$$

where the Schmidt number $S c=2.5$ for naphthalene experiments as in this case.

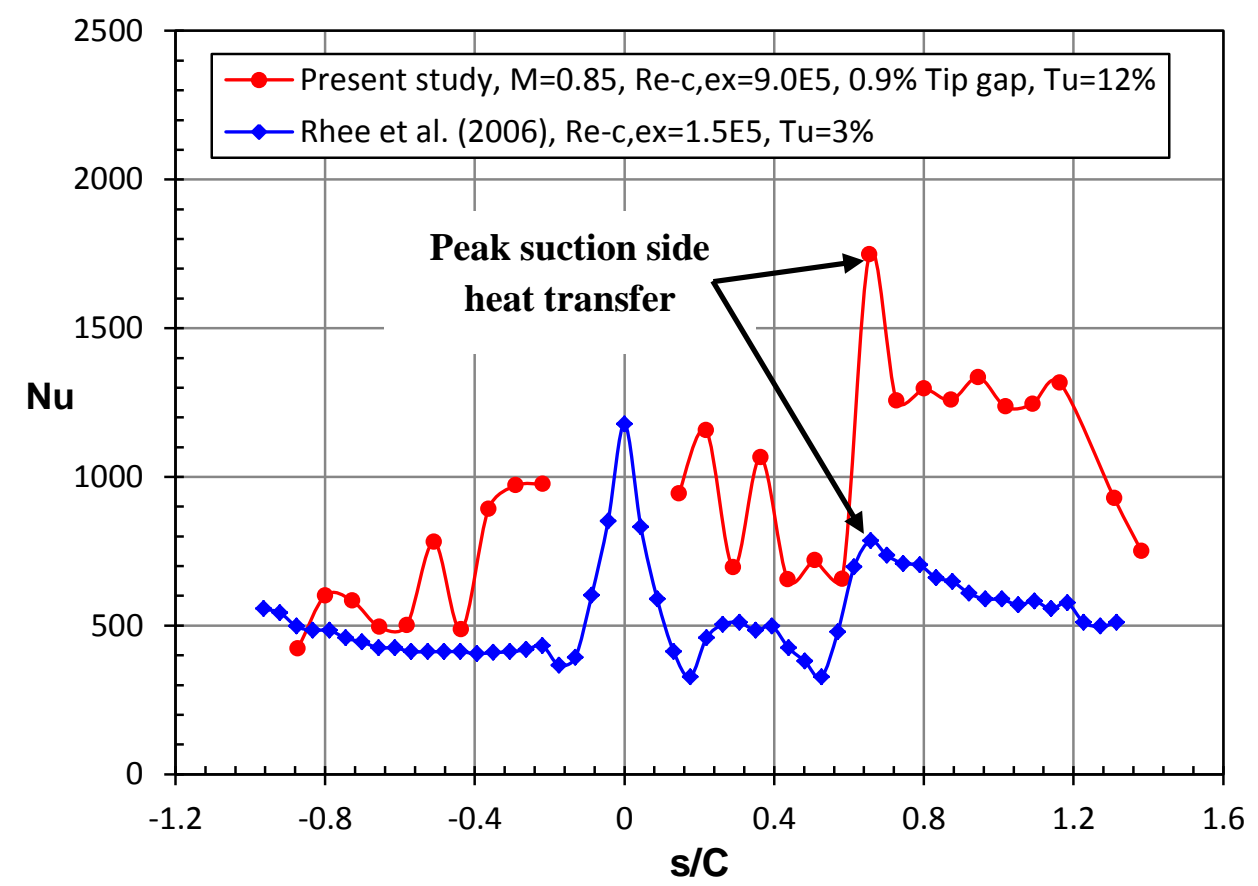

Figure 1.16 Comparison of heat/mass transfer with Rhee et al. [25]

In general, Figure 1.16 shows that the heat transfer characteristics in the present study maintain some similarities with data from Rhee et al., although some discrepancies can be seen. On the suction side, Rhee et al. observed a single peak in heat transfer due to the influence of the 
upstream leakage vortex. The present study on the other hand shows two peaks around this region, $\mathrm{s} / \mathrm{C} \leq 0.44$. The location where the peaks were observed in both tests is approximately the same including that of the local minimum that marks the separation of the upstream vortices from the blade surface. The new high heat transfer region due to the downstream leakage vortex seems to emerge at a similar location, $\mathrm{s} / \mathrm{C} \approx 0.66$, for both tests. For s/C $>0.66$, Rhee et al. observed a steady decline in heat transfer on the suction side. In the present study, heat transfer remains relatively constant between $0.75 \leq \mathrm{s} / \mathrm{C} \leq 1.2$ before dropping steadily towards the trailing edge. Heat transfer levels in the present study appear consistently higher than those from the comparison research work by Rhee et al. The much lower exit Reynolds number condition in the comparison study could be the reason for the decreased heat transfer distribution.

\section{Exit Mach Number Effects on Heat Transfer Distribution}

For a fixed blade tip clearance, the effects of changing the exit Mach numbers below and above the design condition are observed. Nusselt number plots against normalized surface distance are presented to show the heat transfer distribution. Figure 1.17 shows the blade pressure and suction side heat transfer for a $0.9 \%$ tip clearance, based on true chord, with varying exit Mach numbers of $0.74,0.85$ and 1.05 .

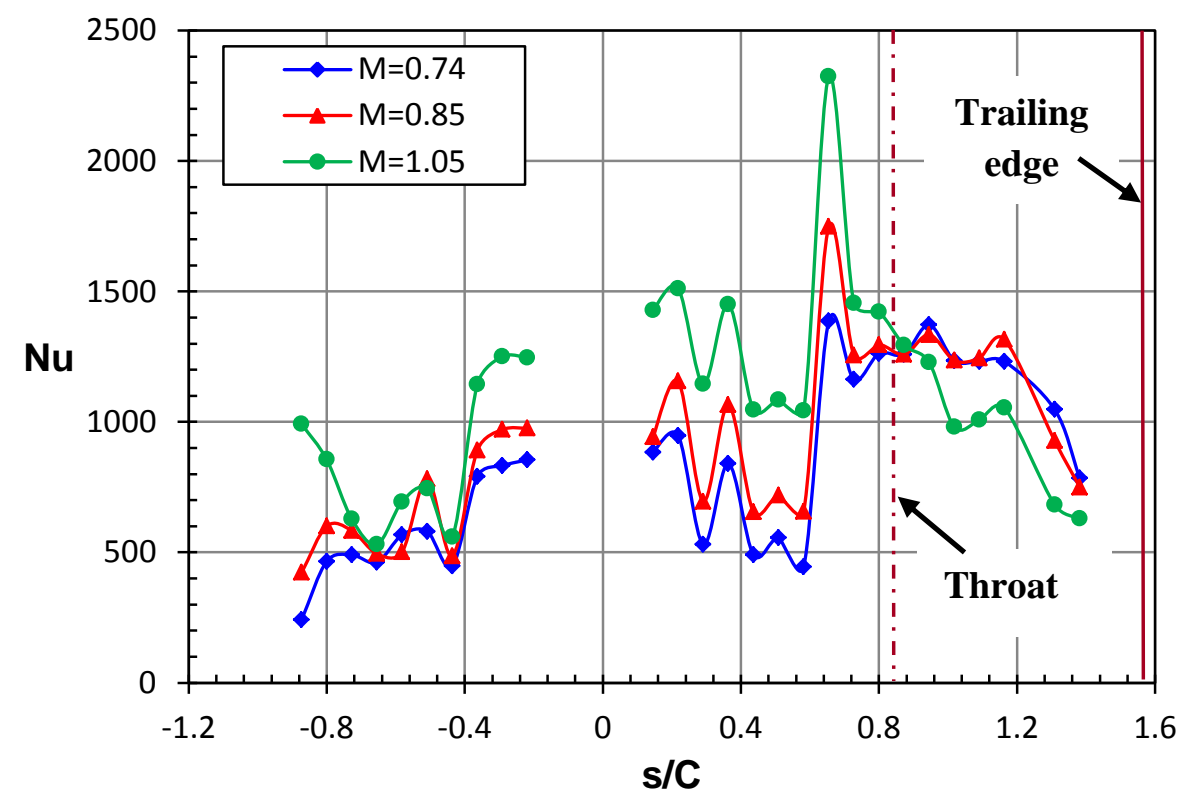

Figure 1.17 Heat transfer distribution for $0.9 \%$ tip clearance 
As indicated by Figure 1.17, an increase in Mach number augments heat transfer on the pressure side and the upstream region of the suction side. The exit Mach 1.05 case shows a rapid drop in heat transfer beyond an s/C of 0.72 . This could be a result of leakage flow separation from the suction side trailing edge region at a high Mach number. The pressure side trailing edge region for the highest Mach number test also indicates a rise in heat transfer.

\section{Tip Clearance Effects on Heat Transfer Distribution}

This section discusses the effects of varying blade tip clearance on heat transfer distribution for a fixed exit Mach number condition. Figure 1.18 provides the heat transfer distributions for each tip clearance at exit Mach 0.85 .

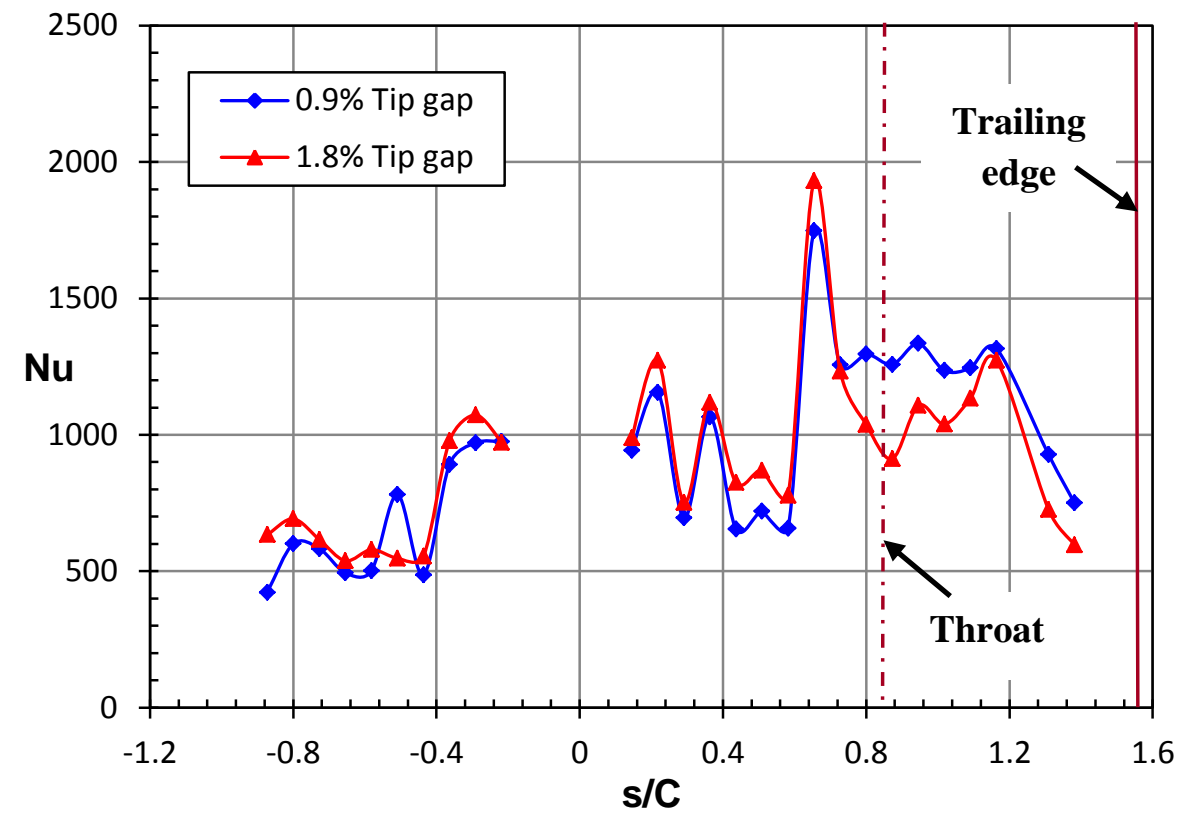

Figure 1.18 Heat transfer distribution at exit Mach number 0.85

An increase in the tip clearance gap seems to augment heat transfer on the near-tip pressure side. The 1.8\% tip gap case shows slightly higher Nusselt number levels on the suction side up until an s/C of 0.73 . The peak Nusselt number at $\mathrm{s} / \mathrm{C}=0.66$ is $15 \%$ higher for the $1.8 \%$ tip gap case compared to the $0.9 \%$ tip clearance test. Between $0.73 \leq \mathrm{s} / \mathrm{C} \leq 1.16$, the Nusselt number level stays fairly constant for the lower tip gap case of $0.9 \%$. For the same range, Nusselt number stays relatively low with the larger tip gap case of $1.8 \%$. This drop in heat transfer could be a result of an enlarged but weakened suction side leakage vortex associated with a larger tip gap. 
At s/C $>1.2$, heat transfer levels sharply drop for both cases. On the pressure side, the local peak Nusselt number at $\mathrm{s} / \mathrm{C}=-0.5$ for the $1.8 \%$ tip gap case is absent for the higher tip gap case.

\section{Conclusions}

Surface oil flow visualization and heat transfer measurements were made on the tip and near-tip region of a turbine blade with a plain tip. Thin film resistance gages were used to obtain heat transfer on the near-tip pressure and suction sides while an infrared thermography technique was used to obtain heat transfer measurements on the tip surface. Accuracy in the IR measurements is limited by the tunnel start up effects on heat transfer coefficients. For future work, other data reduction techniques could be used to account for this unsteadiness caused by the tunnel start up. Directly calculating heat flux before obtaining heat transfer coefficient could be one such option. The features obtained from flow visualization aided in understanding the heat transfer distribution.

The highest heat transfer on the blade tip occurred at the leading edge and along the edge of the pressure side. Relatively low heat transfer was obtained around the suction side leading edge, known as the "sweet spot," and in the small flow separation area on the pressure side edge. Increased leakage flow over a larger tip gap confirmed that increasing the tip clearance gap results in an overall increase in the heat transfer coefficients. An increase in the exit Mach number also resulted in an increased heat transfer on the blade tip.

Heat transfer results on the blade near-tip suction side showed that the leakage flow vortices formed on the suction side dominated the heat transfer distribution. The highest suction side heat transfer was observed at the onset of a strong downstream leakage vortex. Low heat transfer results were obtained towards the trailing edge due to a weakened and enlarged leakage vortex. For the $0.9 \%$ tip gap case, increasing the exit Mach augmented and then decreased, for $\mathrm{s} / \mathrm{C}>0.87$, the suction side heat transfer. Similarly, a larger tip clearance gap of $1.8 \%$ based on engine blade span increased and then decreased the suction side heat transfer for the exit Mach 0.85 case.

The near-tip pressure side heat transfer distribution showed relatively low heat transfer compared to its corresponding suction side heat transfer. The absence of pressure side leakage vortices could be the reason. A local "hot spot" of increased heat transfer was found near the 
midchord section of the near-tip pressure side. An increased tip clearance gap and exit Mach seemed to slightly augment heat transfer on the near-tip pressure side region.

\section{Acknowledgment}

This work was sponsored by Solar Turbines Inc.

\section{References}

[1] Sjolander, S.A. and Cao, D., 1995, "Measurements of the flow in an idealized turbine tip gap,” ASME J. Turbomach., 117, pp. 578-584.

[2] Nicole L. Key and Tony Arts, 2006, "Comparison of Turbine Tip Leakage Flow for Flat Tip and Squealer Tip Geometries at High-Speed Conditions," ASME J. Turbomach., 128, pp. 213220.

[3] Booth,T. C., Dodge, P. R., and Hepworth, H. K., 1982, "Rotor-tip Leakage. I. Basic

Methodology,".ASME Journal of Engineering for Gas Turbines and Power, 104, pp. 154-161.

[4] Mayle, R. E., and Metzger, D. E., 1982, "Heat Transfer at the Tip of an Unshrouded Turbine Blade," Proceedings of the Seventh International Heat Transfer Conference, Hemisphere, New York, pp. 87-92.

[5] Yang, D., Yu, X. and Feng, Z., 2010, "Investigation of Leakage Flow and Heat Transfer in a Gas Turbine Blade Tip with Emphasis on the Effect of Rotation," ASME J. Turbomach., 132, 041010

[6] Azad, G. M. S., Han, J. C., and Boyle, R., 2000, "Heat Transfer and Pressure Distributions on the Squealer Tip of a Gas Turbine Blade," ASME J. Turbomach., 122, pp. 725-732.

[7] Kwak, J.S., and Han, J.C., 2003, "Heat Transfer Coefficients of a Turbine Blade-Tip and Near-Tip Regions," AIAA Journal of Thermophysics and Heat Transfer, 17, pp. 297-303.

[8] Zhang, Q., O'Dowd, D. O., He, L., Wheeler, A. P. S., Ligrani, P. M., and Cheong, B. C. Y., 2011, "Overtip Shock Wave Structure and Its Impact on Turbine Blade Tip Heat Transfer,” ASME J. Turbomach., 133(4), p. 041001

[9] Holmberg, D.G., and Diller, T.E., 2005, "Simultaneous Heat Flux and Velocity Measurements in a Transonic Turbine Cascade," ASME J. Turbomach., 127, pp. 502-506. 
[10] Nix, A.C., Diller, T.E., and Ng, W.F., 2007, "Experimental Measurements and Modeling of the Effects of Large-Scale Freestream Turbulence on Heat Transfer," ASME J. Turbomach., 129, pp. 542-550.

[11] Smith, D.E., Bubb, J.V., Popp, O., Grabowski, H.C., Diller, T.E. Schetz, J.A. and Ng. W.F., 2000, "Investigation of Heat Transfer in a Film Cooled Transonic Turbine Cascade, Part I: Steady Heat Transfer," ASME 2000-GT-202.

[12] Popp, O., Smith, D.E., Bubb, J.V., Grabowski, H.C., Diller, T.E. Schetz, J.A. and Ng. W.F., 2000, "Investigation of Heat Transfer in a Film Cooled Transonic Turbine Cascade, Part II:

Unsteady Heat Transfer," ASME 2000-GT-203.

[13] Carullo, J. S., Nasir, S., Cress, R. D., Ng, W. F., Thole, K. A., Zhang, L. J., and Moon, H. K., 2011, "The Effects of Freestream Turbulence, Turbulence Length Scale, and Exit Reynolds Number on Turbine Blade Heat Transfer in a Transonic Cascade," ASME J. Turbomach., 133, 011030

[14] Nasir, S., Carullo, J.S., Ng, W.F., Thole, K.A., Wu, H., Zhang, L.J., and Moon, H.K., 2009, "Effects of Large Scale High Freestream Turbulence, and Exit Reynolds Number on Turbine Vane Heat Transfer in a Transonic Cascade," ASME J. Turbomach., 131, 021021

[15] Ekkad, S. V., Ou, S., and Rivir, R. B., 2004, “A Transient Infrared Thermography Method for Simultaneous Film Cooling Effectiveness and Heat Transfer Coefficient Measurements From a Single Test," ASME J. Turbomach., 126, pp. 546-553.

[16] Schultz, D.L., and Jones, T.V., 1973, "Heat Transfer Measurements in Short Duration Hypersonic Facilities," AGARD AG-165.

[17] Doorly, J.E., and Oldfield, M.L.G., 1987, "The Theory of Advanced Multi-Layer Thin Film Heat Transfer Gages," Intl. J. Heat and Mass Transfer, 30, pp. 1159-1168.

[18] Dunn., M.G., 1995, “The Thin-Film Gage," Von Karman Institute for Fluid Dynamics, Lecture Series 1995-01.

[19] Joe, C.R., 1997, "Unsteady Heat Transfer on the Turbine Research Facility at Wright Laboratory," Ph.D. Dissertation, Syracuse University.

[20] Cress, R.D., 2006, "Turbine Blade Heat Transfer Measurements in a Transonic Flow Using Thin Film Gages,” Master's Thesis, Virginia Polytechnic Institute and State University.

[21] Moffat, R. J., 1988, “Describing Uncertainties in Experimental Results," Exp. Thermal and Fluid Science, 1988, pp. 3-17.

[22] Bunker, R. S., Bailey, J. C., and Ameri, A. A., 1999, "Heat Transfer and Flow on the First Stage Blade Tip of a Power Generation Gas Turbine: Part 1-Experimental Results," ASME J. Turbomach., 122, pp. 263-271. 
[23] Nasir, H., Ekkad, S.V., Kontrovitz, D.M., Bunker, R.S., Prakash, C., 2004, "Effect of tip gap and squealer geometry on detailed heat transfer measurements over a high pressure turbine rotor blade tip," ASME J. Turbomach., 126, pp. 221-228.

[24] Moore, J., and Tilton, J. S., 1988, "Tip Leakage Flow in a Linear Turbine Cascade," ASME J. Turbomach., 110, pp. 18-26.

[25] Rhee, D. H., and Cho, H. H., 2006, "Local Heat/Mass Transfer Characteristics on Rotating Blade With Flat Tip in a Low Speed Annular Cascade_-Part I: Near-Tip Surface," ASME J. Turbomach., 128, pp. 96-109. 


\section{Appendix A Cascade Setup}

\section{Test Section Setup}

\section{Macor Tip of the Center Blade}

Figure A.1 shows the Macor tip of the center blade, where the thin film gages (TFG) were mounted, at $94 \%$ of engine blade span. This Macor blade tip, $38 \mathrm{~mm}$ (1.5 in.) in span, was mounted onto a stainless steel blade section ( $114 \mathrm{~mm}$ or $4.5 \mathrm{in}$. in span) to make up the entire 6 in. span of the test section. The mounting of the Macor tip to the stainless steel blade section is to provide structural integrity for the cantilever blade with the tip gap at the end of the Macor tip section.

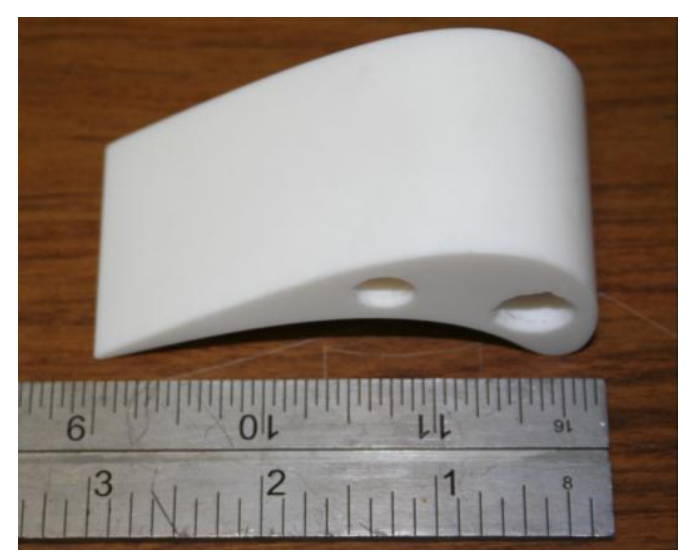

Figure A.1 Macor tip of the test blade

\section{Mounting and Adjustment of Blade Tip Clearance in the Test Section}

Figure A.2 shows the assembly of the circular window of one side of the wind tunnel test section. The cascade includes seven blades. The center blade, as previously mentioned, is made up of both stainless steel and Macor. The two adjacent blades are made entirely of stainless steel. The center blade as well as the adjacent blades are mounted on the same insert plate (Aluminum material), as shown in Figure A.2. Therefore, the center and the adjacent blades are moved together as a whole set with the insert plate, to create different tip gaps on the circular window mounted on the other side of the tunnel's sidewall. Only the center three blades are cantilevered to create the tip gaps. The remaining four blades will be mounted with pins on circular windows on both sides of the tunnel. 


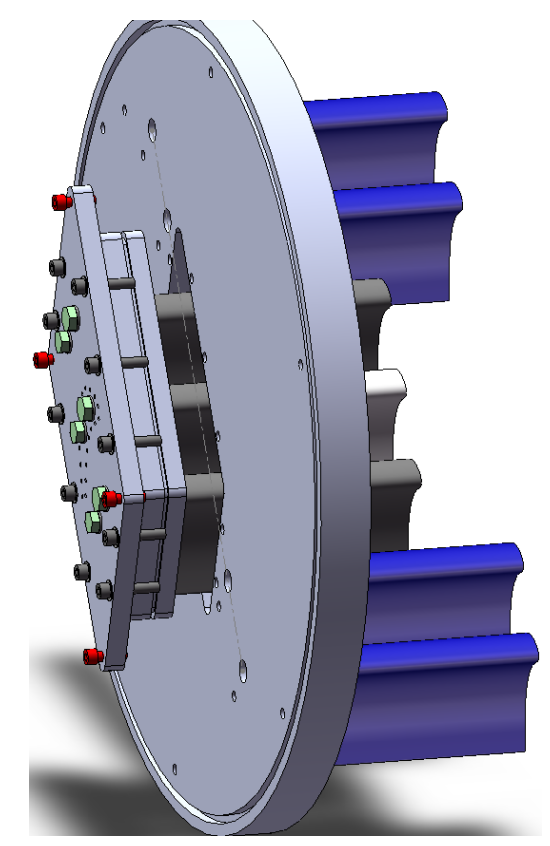

Figure A. 2 Mounting and adjustment of tip clearance in the test section

As shown in Figure A.2, at the four corners of the aluminum insert plate, there are four screws that are used to set a precise tip gap between the blade tip and the other side wall. As the three central blades are adjusted with the insert plate, different tip clearances are achieved. These four screws also ensure that the blade tip surfaces are parallel to the opposite window that simulates the tip casing. Once the adjustment of the tip clearance is done, the mounting bolts are tightened to hold the insert plate in position. Figure A.3 shows the instrumented blade in the test section during installation.

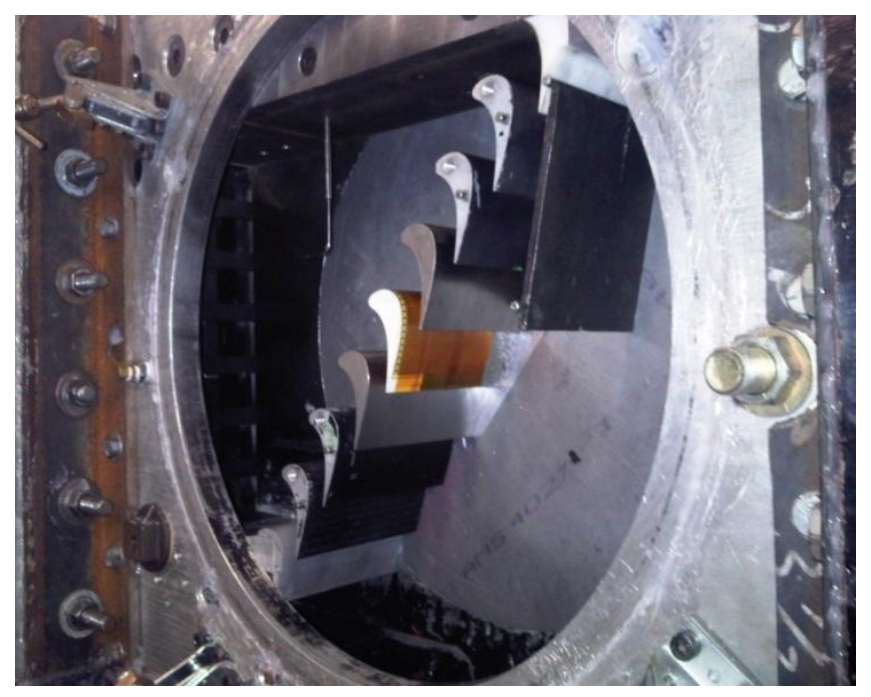

Figure A.3 Picture of test section with instrumented blade in place 


\section{Appendix B Exit Mach Number Distribution}

\section{Pressure Tap Layout}

Static pressure taps were installed on the end wall of the cascade at the exit of the passages. Eight (8) wall taps were placed approximately $0.5 \mathrm{C}$ downstream of the cascade and spaced $1 \mathrm{in}$. apart. These end wall taps were used, in conjunction with the upstream total pressure, to obtain the test exit Mach number. In addition, the wall taps were also used to measure the uniformity of the exit flows. Figure B.1 shows the layout of the exit static taps in the cascade. A picture of the pressure taps instrumented on the blade are provided in Figure B-2.

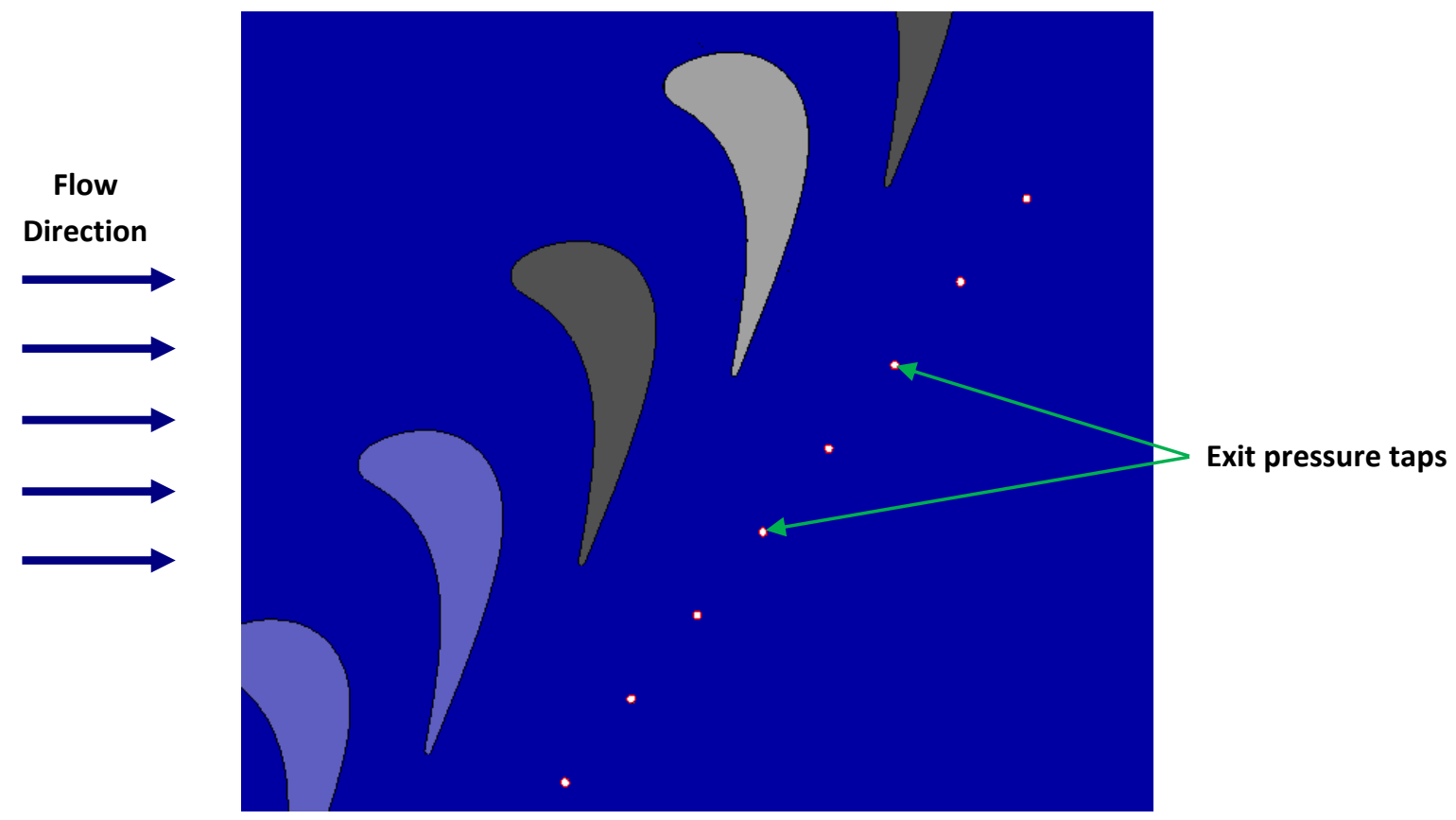

Figure B.1 Schematic showing the pressure tap locations on the endwall

\section{Static Pressure Measurement Technique}

A Model 98RK Net Scanner System was used to collect the static pressure distribution on the blade during the run. Pressure data throughout this study was collected at a frequency of 10 Hz. The static pressure data from each pressure tap was converted into a pressure ratio using the total pressure of the flow measured with a Pitot-static probe located upstream of the cascade 
inlet. With the pressure ratio, the local exit Mach number could then be calculated by using equation B.1,

$$
M a_{2}=\sqrt{\frac{2}{\gamma-1} \cdot\left(P R^{\frac{\gamma-1}{\gamma}}-1\right)}
$$

where PR is the pressure ratio and $\gamma$ is the specific heat ratio of air. It was assumed that there were no losses through the blade passages, so the total pressure remained constant. Once the Mach numbers were calculated, the data was averaged over 10 seconds of the run where the exit Mach number was steady. Results from all pressure taps were then averaged to obtain a single value of the exit Mach number for any given run.

\section{Blade Passage Periodicity}

It is important that the pressure distribution through each passage remain fairly consistent. A tailboard, placed immediately downstream of the test section, was used to ensure that periodic flow is obtained through the blade passages in the test section.

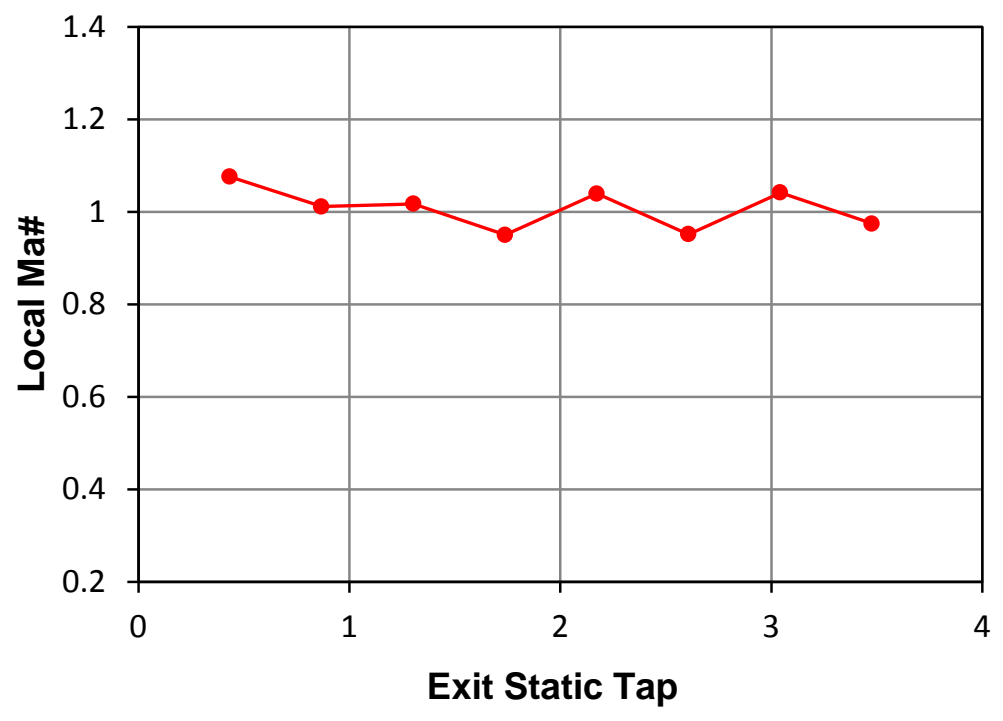

Figure B. 2 Local exit Mach comparison for a Mach 1 test

Figure B.2 shows the exit Mach number values as calculated from the exit pressure tap readings. For the test shown, it is clear that the flow is periodic as evident by the relatively steady Mach number values across the blade passages. 


\section{Appendix C Infrared (IR) Technique}

In order to capture the blade tip surface temperature during the experiments, an infrared (IR) camera was put in place for this purpose. This camera is capable of recording the surface temperature of a body, the blade tip in this case, based on the black body emission that is radiated from the surface of that body. The camera views the blade tip through a special IR transparent window made of Zinc Selenide. Figure C.1 shows the IR window setup.

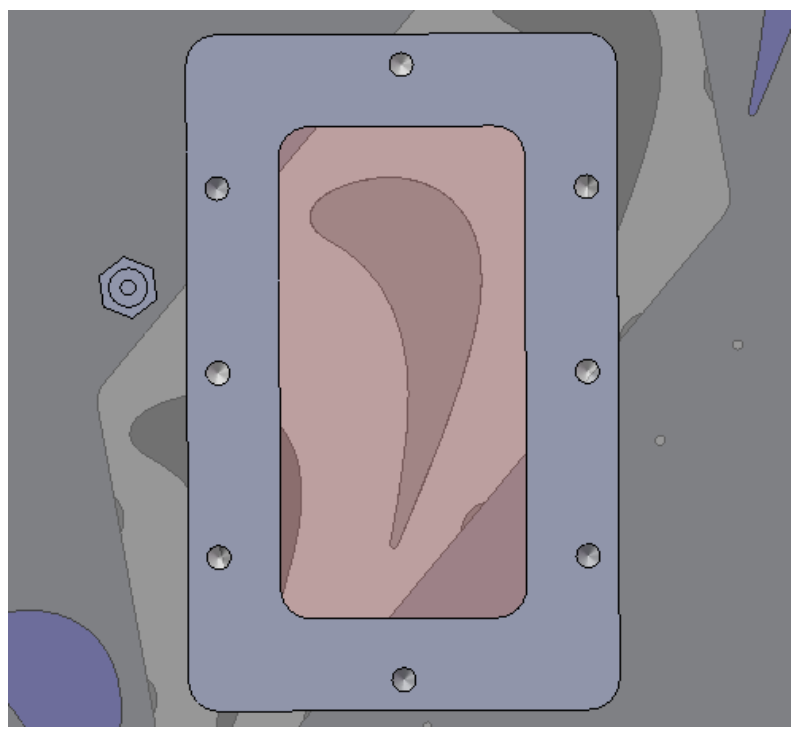

Figure C.1 IR window

To increase the IR transmissivity, an anti-reflective coating was added onto the Zinc Selenide window. The entire test section was painted black, in order to reduce reflected radiation and increase the blade tip emissivity. Surface thermocouples were positioned in situ for IR camera calibration purposes. During calibration, a sheet thermocouple was mounted on the tip surface where it was visible through the transparent $\mathrm{ZnSe}$ window. A digital thermocouple reader was then used to read the blade tip temperature while an external heater was used to blow hot air over the surface. Simultaneously, certain IR camera properties were adjusted to make the IR temperature match that from the thermocouple on the exact spot where the thermocouple was placed. A wide temperature range was tested to ensure that the temperature data from the IR camera is accurate. 


\section{Heat Transfer Data Reduction}

The equation for transient heat conduction through a semi-infinite solid was solved using a convective boundary condition. The solution is well established and was earlier mentioned in this work in the previous section as Equation 1. The solution is again stated in Equation C.1

$$
\frac{T_{w}-T_{i n i t, w}}{T_{\infty}-T_{i n i t, w}}=1-\left[\exp \left(\frac{h^{2} \alpha t}{k^{2}}\right)\right]\left[\operatorname{erfc}\left(\frac{h \sqrt{\alpha t}}{k}\right)\right]
$$

where $h$ is the only unknown. This solution makes the simplification that $T_{\text {aw }}$ is equivalent to $T_{\infty}$. To solve for $h$, the measured wall temperature history is curve fit to the solution derived above using a Matlab program and the fminsearch function to minimize error in the curve fit. More information on this procedure is given by Reagle [G-1]. A sample curve fit is presented in Figure C.2. The data reduction window for this method is $0-5 \mathrm{~s}$.

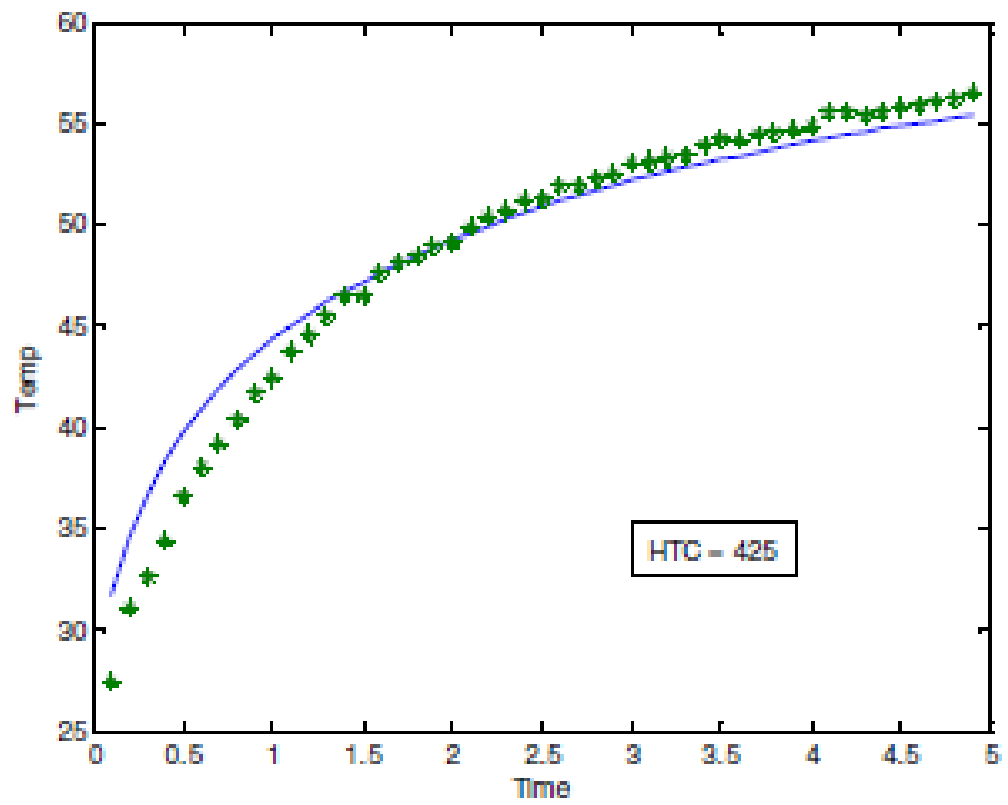

Figure C. 2 Example curvefit of blade IR data 


\section{Appendix D Thin Film Gage Overview Operating Principles}

The thin film gages used consist of a platinum sensor and copper leads on a thin Upilex sheet. The gages are attached to the surface of the blade and indirectly measure the heat flux into the blade. The resistances of the gages change along with changes in surface temperature. The resulting two-layered system can be seen in Figure D.1.

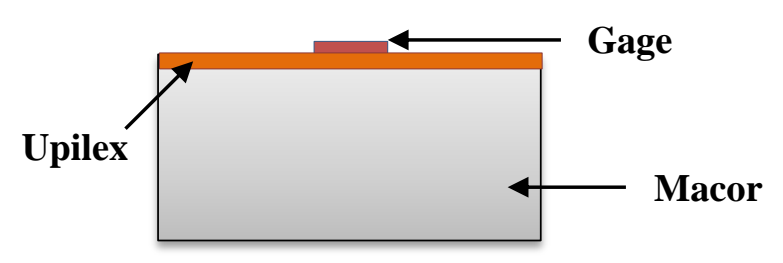

Figure D. 1 Diagram of multi-layered gage design (not drawn to scale)

The gages are supplied a constant current by a Wheatstone bridge so that a voltage change, rather than a direct measurement of resistance change, can be recorded. This voltage change is proportional to the resistance change that is caused by a surface temperature change. From this voltage, the surface temperature time resolved history is calculated. That temperature is then used to solve for heat flux into the blade.

\section{Gage Installation and Calibration}

Before mounting the gauges on a low thermally conductive Macor $(k=1.46 \mathrm{~W} / \mathrm{mK})$ blade, the gage sheet was cut to the appropriate size, and the Macor portion of the blade was thoroughly cleaned. The platinum sections of the gauges were placed at a distance of $0.18 \mathrm{in}$. from the blade tip edge ( $94 \%$ of engine blade span). The gage sheet was carefully adhered to the pressure and suction surfaces. Air bubbles were eliminated beneath the sheet by using a soft paper towel to apply the sheet from the stagnation region toward the trailing edge. Once the gauges were installed on the Macor blade, the lead wires were soldered to the copper leads. The resistance of each gauge was measured and recorded after soldering of the lead wires. A layer of JB Weld was applied over all of the solder joints to make sure that the lead wires would not detach from the gauges due to the high speed flow during a test run. The JB Weld is 
nonconductive and does effect the operation of the gauge. Figure D.2 shows a picture of the blade with the thin film gages installed.

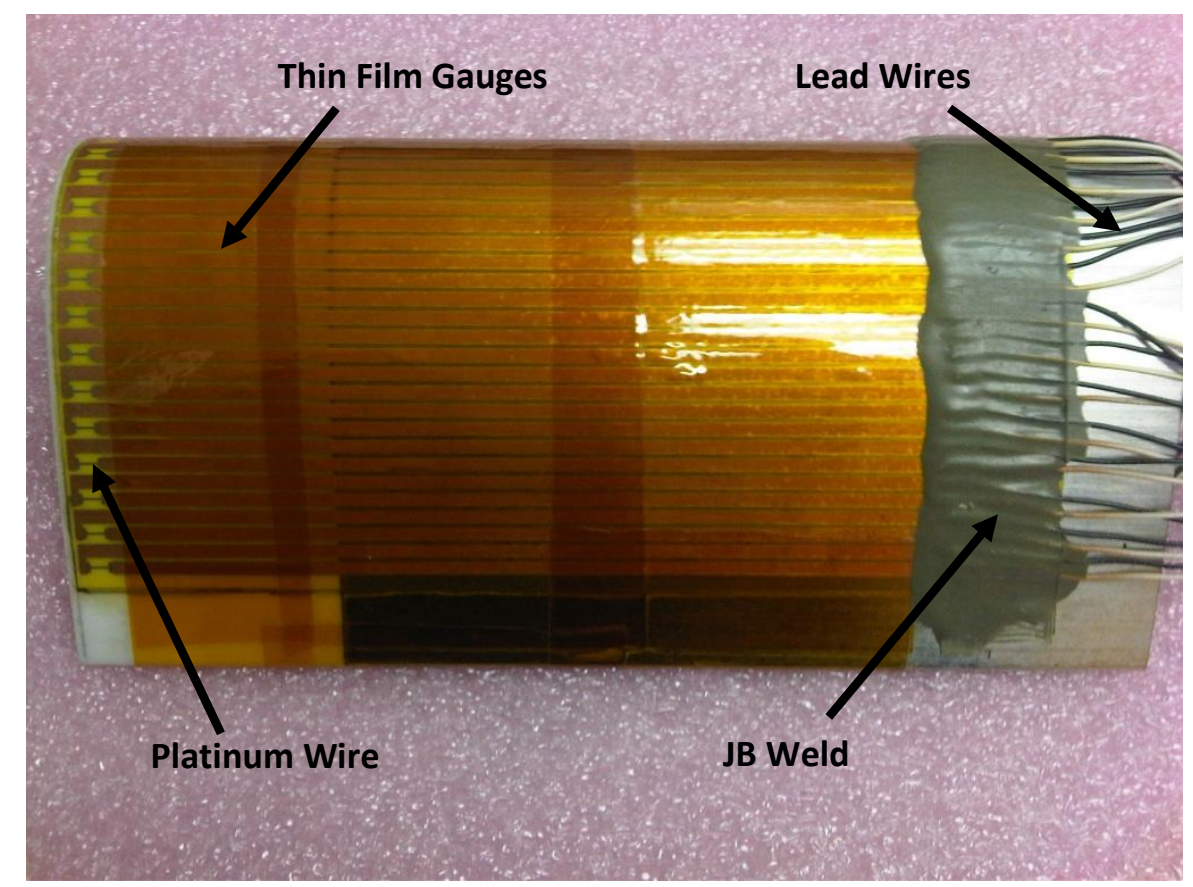

Figure D. 2 Picture of test blade with thin film gage installed

Once lead wires were soldered, the thin film gauges were calibrated for temperature coefficient of resistivity in a low temperature oven over the range of temperatures encountered during a tunnel test. The calibration consisted of increasing the temperature of the oven in five discrete steps from ambient temperature up to $\sim 85^{\circ} \mathrm{C}$ and recording the resistance of each gauge at that temperature. The temperature of the oven was then decreased back to ambient in four discrete steps and again the resistance of each gauge was recorded at each temperature. Typically 2 hours was allowed to pass between calibration points so that the oven could reach a steady temperature and to ensure that the blade was thermally soaked. Calibration points were recorded as the temperature increased and decreased to check for gauge hysteresis. During the calibration phase, a few issues that must be considered came about. Some gages were exhibiting unusual behavior during the calibration. Those gages would show a steady linear calibration between resistance and temperature while heating the gage up, but then once the gage reached the maximum temperature or as the gage was cooling down to the lower temperatures, there would be a significant shift in resistance. Figure D.3 shows this trend for one of the gages. 


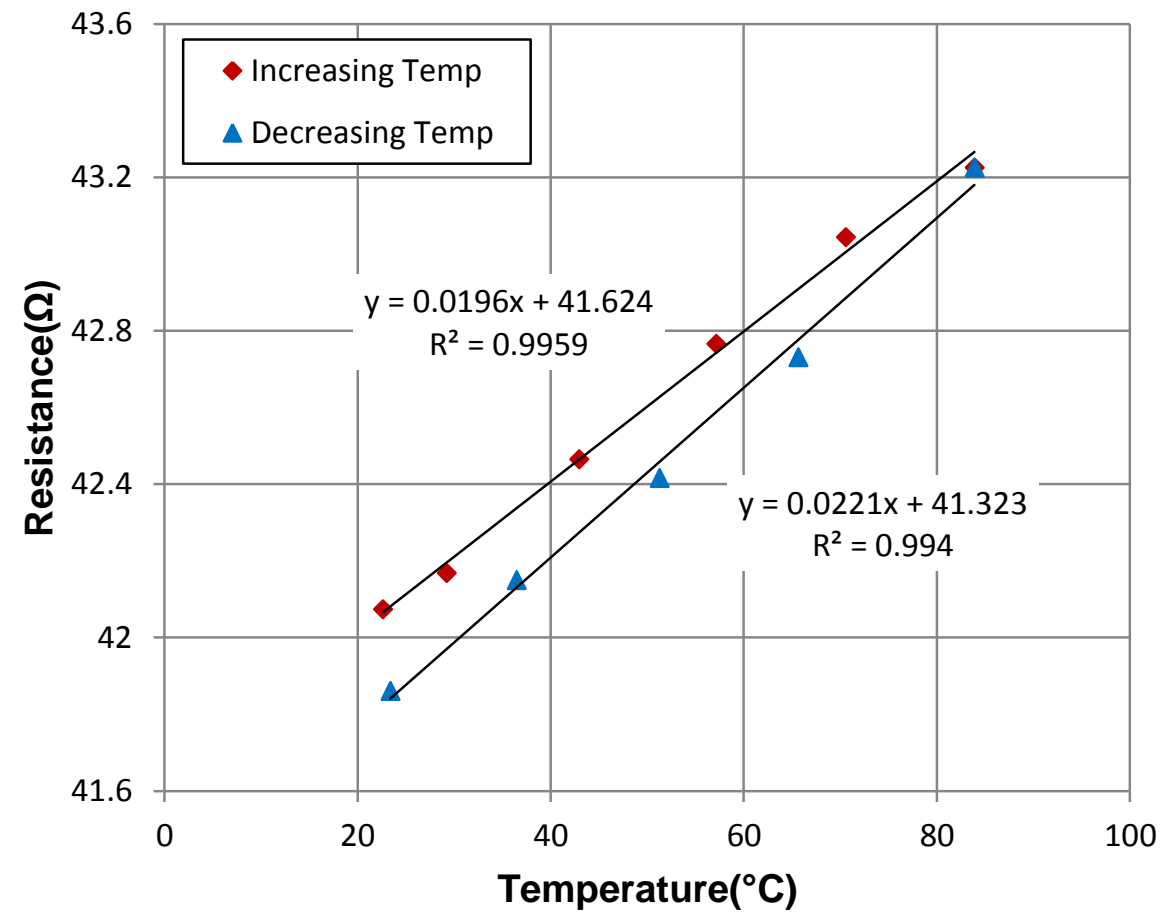

Figure D. 3 Calibration of a single gage that exhibited unstable behavior

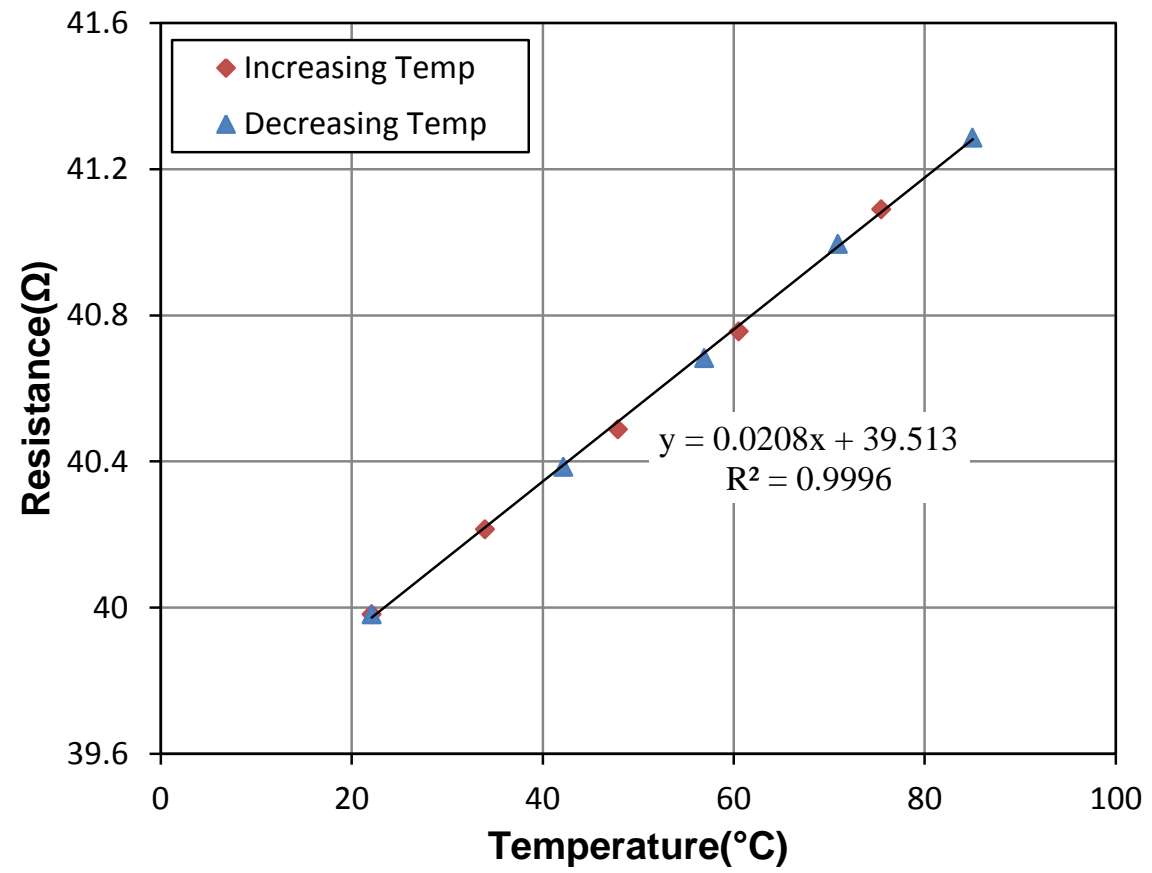

Figure D. 4 Calibration of a single gage that exhibited stable behavior 
The reason for this sudden decrease in resistance seemed unclear at first until the issue was thought out more thoroughly. It turns out that the gages needed to be heated and cooled through several cycles in order to stabilize their properties. To this end, the gages were placed in the oven and the temperature raised to about $90^{\circ} \mathrm{C}$ before cooled subsequently. The repeated heating and cooling provided an annealing effect on the gages. A more refined linear calibration fit for one of the gages is provided in Figure D.4.

As previously mentioned, each thin film gauge acts as one arm of an individual Wheatstone bridge circuit. The constant current supplied by the balanced Wheatstone bridge circuit ensures that a voltage change, rather than a direct measurement of resistance change, can be recorded by National Instruments (NI) data acquisition system as the blade surface temperature changes. Details of the Wheatstone bridge circuit used in this experiment have been previously recorded by Cress [G-2]. Figure D.5 provides a schematic of the Wheatstone bridge circuit of the thin film gauges.

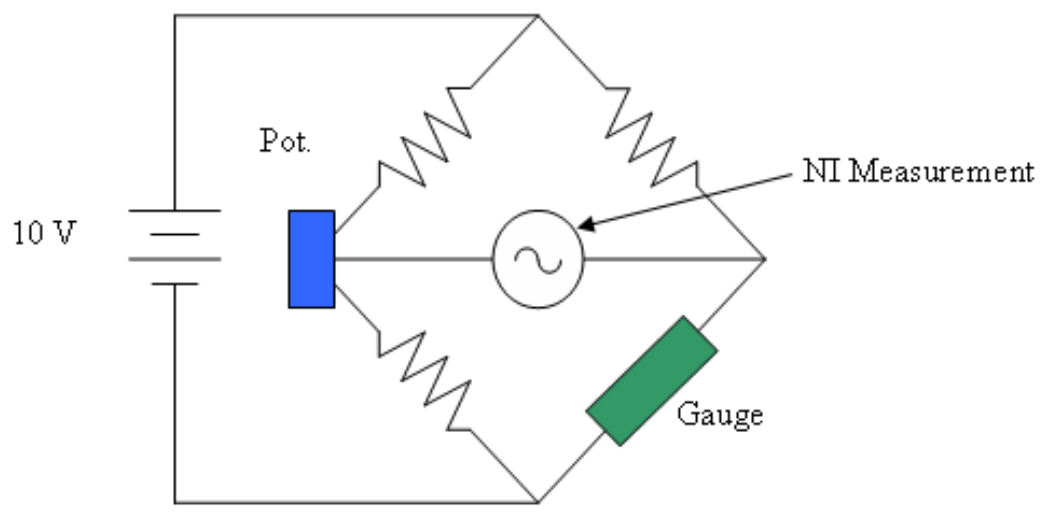

Figure D. 5 Schematic of the Wheatstone bridge circuit for the thin film gauges

\section{Heat Transfer Gage Layout}

The gages were used to capture heat transfer results near the blade tip at about $94 \%$ of engine blade span.

A single sheet with thirty two (32) equally-spaced thin film gages was installed towards the trailing edge suction surface, wrapped around the leading edge, and then affixed unto the 
pressure side. Overall, the layout consists of twelve (12) gages on the pressure surface, two (2) gages around the leading edge and twenty (20) gages on the suction surface. Figure D-6 shows the gage locations chord-wise, around the blade pressure and suction sides. During the tunnel run, few gages mostly around the leading edge, were lost and these gages have been omitted from Figure D.6.

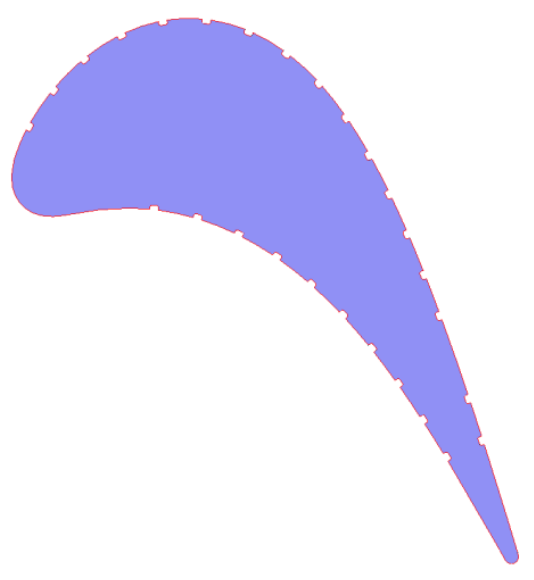

Figure D. 6 Blade profile showing thin film gage locations

\section{Thin Film Gauge Data Collection}

Prior to the first run of a day, the resistances of the gages were measured and recorded for eventual data reduction purposes. Then the potentiometers in the Wheatstone bridges were adjusted to balance the bridges (i.e. zero the output voltage).

The gages were connected to a National Instruments SCXI data acquisition system. The SCXI system consisted of an SCXI-1001 12 module chassis, an SCXI-1600 16-bit data acquisition board, and four SCXI-1120 8-channel input modules. The SCXI-1120 modules have built in filtering and amplification that were used in recording the heat flux gage data. The heat transfer data was sampled at a rate of $1 \mathrm{kHz}$ with a gain of 500 .

Upstream and blade surface temperature were also obtained using thermocouples attached to the same data acquisition equipment. The thermocouples were similarly sampled at 1 $\mathrm{kHz}$. 


\section{Heat Transfer Data Reduction}

The output from the thin film gauges via the Wheatstone bridge comes in the form of voltage versus time. Each recorded thin film gauge voltage is then converted into surface temperature using the basic Wheatstone bridge operating principles and gage calibration. Next, the normal heat flux for each gauge is calculated by using a finite-difference code developed by Cress [G-2]. The finite-difference code uses the surface temperature history of the gauge as a boundary condition and solves the one-dimensional, transient heat conduction equation using a semi-infinite boundary condition. The initial temperature of the blade is determined via thermocouples installed on the Macor section of the blade surface prior to the run. 1-D semiinfinite heat conduction can be assumed since the gauges are installed on the low thermally conductive Macor portion of the test blade. Figure D.7 shows the surface temperature and the normal heat flux into the blade for a sample gauge during a run.

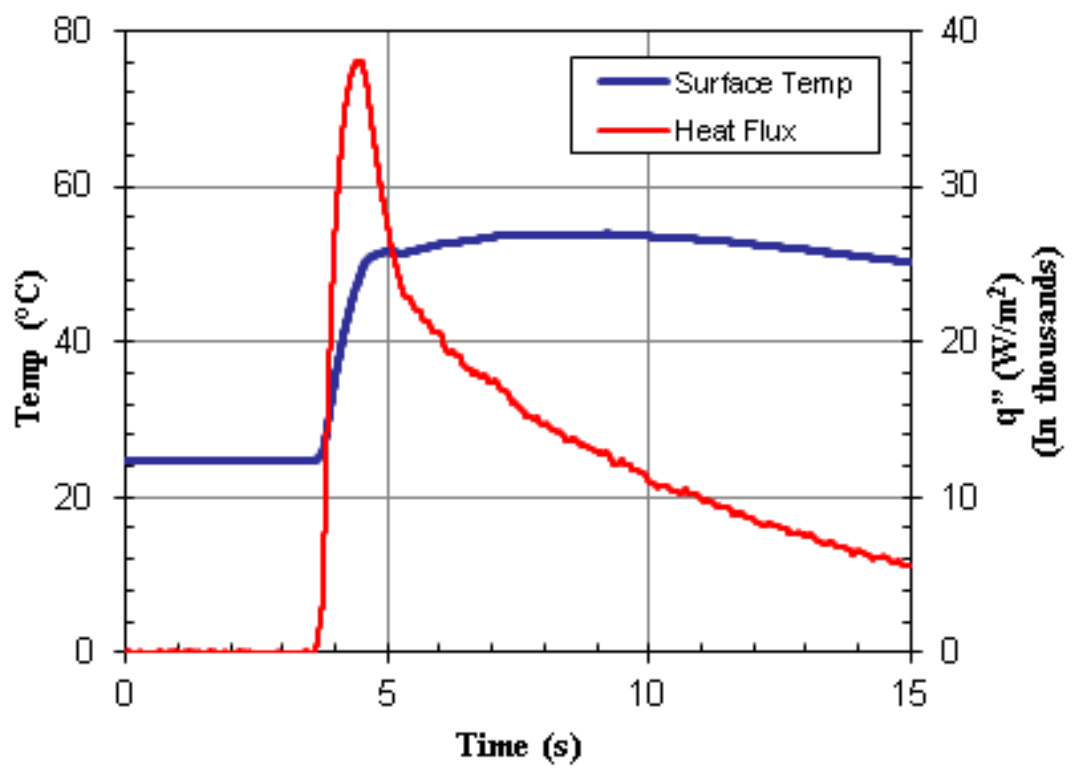

Figure D. 7 Sample surface temperature and heat flux histories

Once heat flux is calculated, the heat transfer coefficient can be found using the least squares linear regression fit of the following equation.

$$
q^{\prime \prime}=h\left(T_{\infty}-T_{w}\right)-h\left(T_{\infty}-T_{a w}\right)
$$

where $q$ " is calculated with the single-sided thin film gauge. 
Using Equation D.1, we can determine $h$ and $T_{a w}$ by plotting $q$ ” versus $\left(T_{\infty}-T_{w}\right)$. The result gives us a line of the form $y=m x+b$, where the slope of the line is the heat transfer coefficient and the $\mathrm{x}$-intercept of the line is $\left(T_{\infty}-T_{a w}\right)$. The slope is determined using a least squares linear regression fit of the plotted data. More explanation of this technique can be seen in Smith et al. [G-3] and Popp et al. [G-4]. A sample plot showing this technique is presented in Figure D.8. The heat transfer coefficient can then be nondimensionalized by calculating the Nusselt number using,

$$
N u=\frac{h \cdot C}{k}
$$

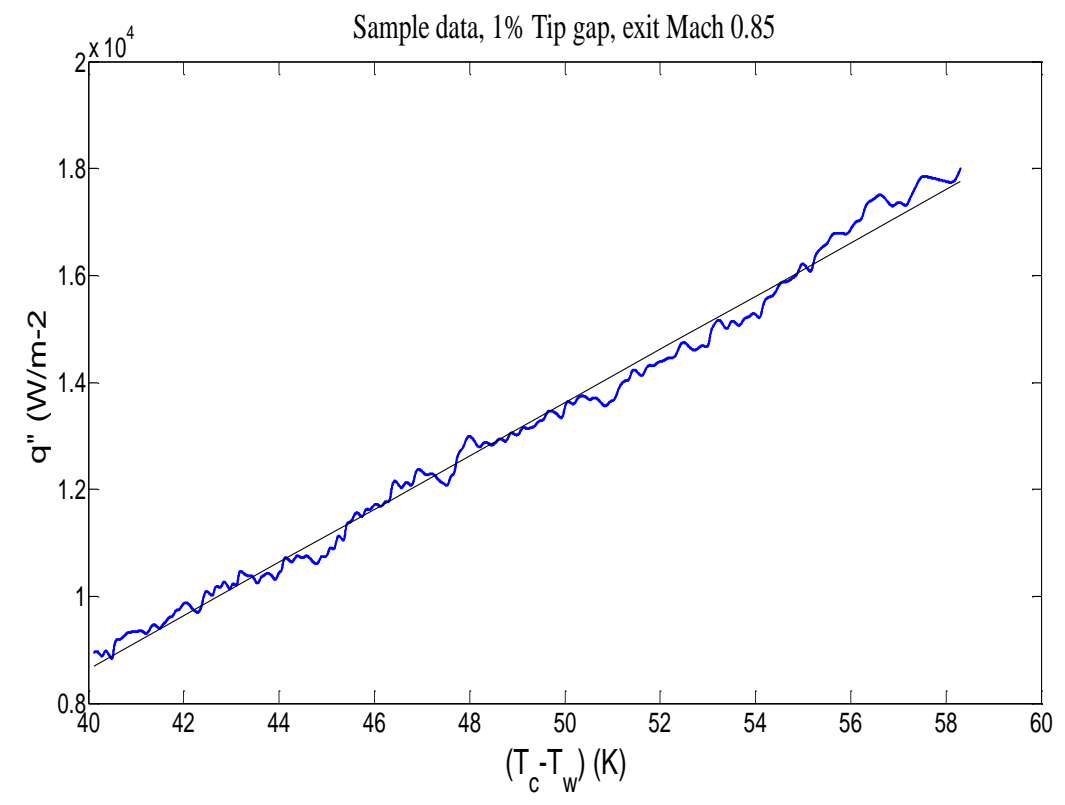

Figure D.8 Determination of heat transfer coefficient

Note that heat transfer data using both the IR thermography technique, as described previously, and the thin film gages were obtained at the same time during these tests. 


\section{Appendix E Additional Results}

\section{Flow Visualization}

Figures E.1 and E.2 show the surface flow visualization on the blade near-tip suction side and tip surface respectively. The results were obtained at exit Mach 1.05 with $0.9 \%$ tip clearance gap.

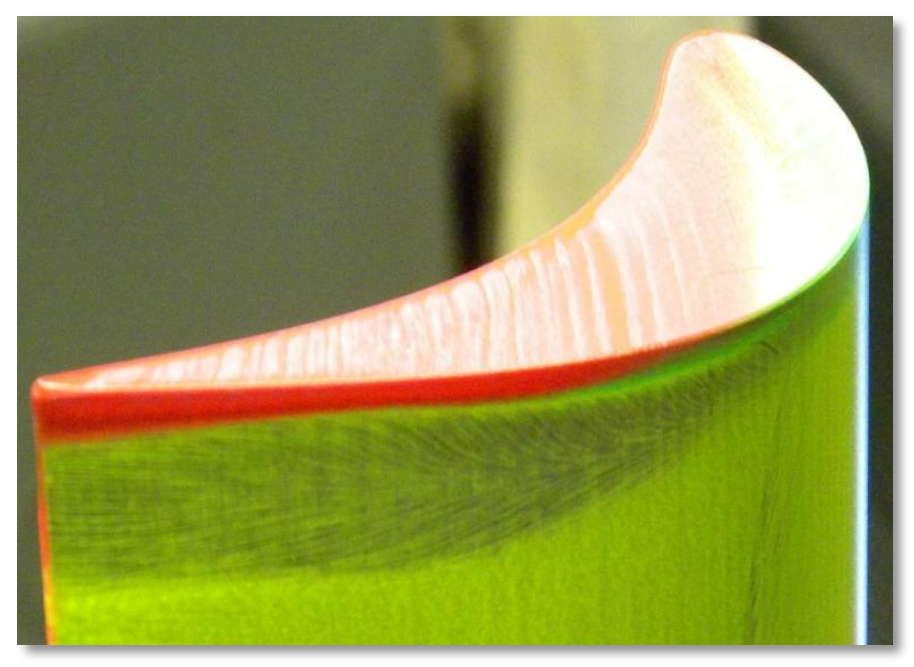

Figure E. 1 Suction side flow visualization at $\mathrm{M}=1.05$ with a tip clearance of $0.9 \%$

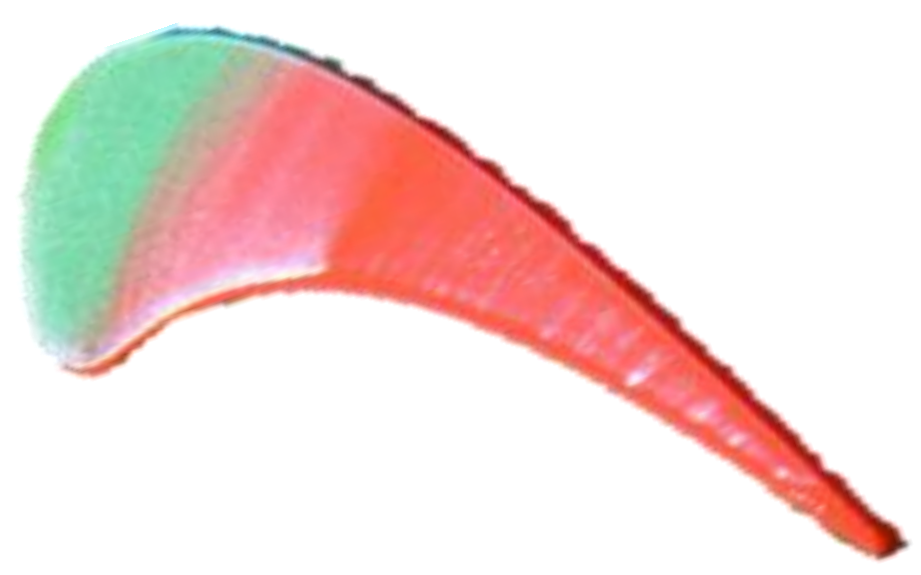

Figure E. 2 Blade tip flow visualization at $\mathrm{M}=1.05$ with a tip clearance of $0.9 \%$ 


\section{Blade Tip Heat Transfer \\ Camber Line Heat Transfer Coefficient}

Figures E. 3 and E.4 detail the averaged heat transfer coefficients on the blade tip at a fixed exit Mach number of 0.85 , and for a given tip clearance of $0.9 \%$ based on engine blade span respectively. The local heat transfer coefficients are obtained along the mean camber line of the blade tip surface. It can be seen from Figure E. 3 that an increase in the tip clearance gap results in an overall increase in the heat transfer coefficients. In the same vein, an increase in the Mach number shows an increase in heat transfer as shown in Figure E.4.

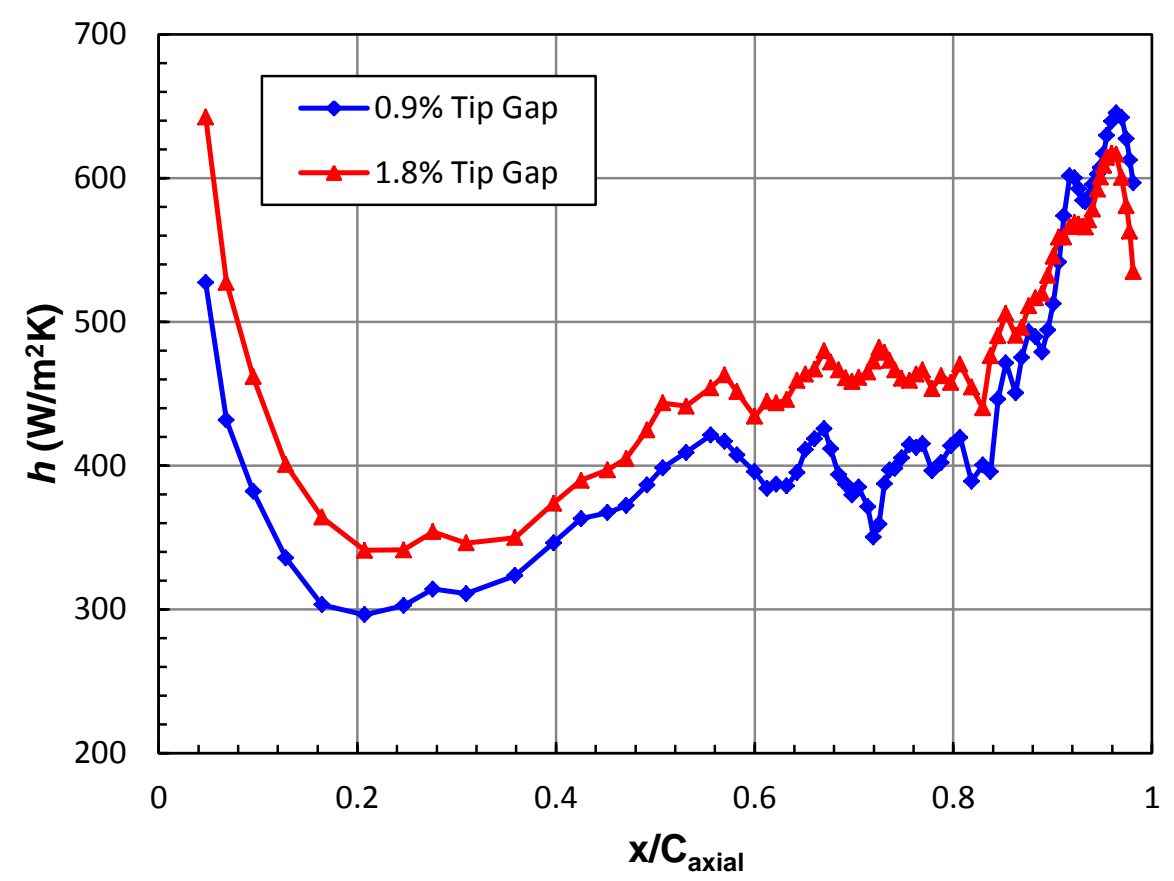

Figure E. 3 Measured heat transfer coefficients along the camber line of the blade tip at exit Mach 0.85 


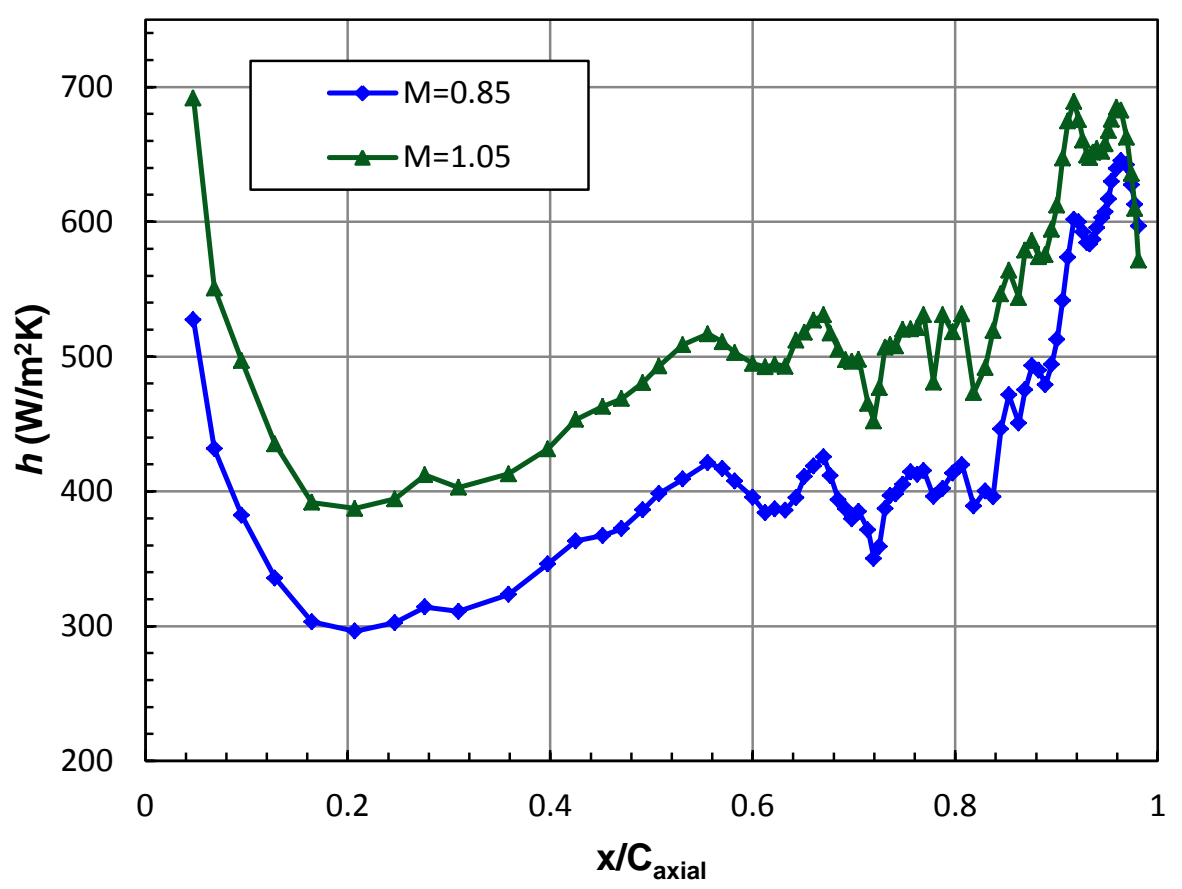

Figure E . 4 Measured heat transfer coefficients along the camber line of the blade tip at $0.9 \%$ tip clearance

\section{Near-Tip Heat Transfer}

\section{Tip Clearance Effects on Heat Transfer Distribution}

Figure E.5 provides the heat transfer distributions for each tip clearance at exit Mach 0.7. An increase in the tip clearance gap seems to augment heat transfer on the near-tip pressure side.

The Nusselt number levels at exit Mach 0.7 for both tip clearances are largely similar on the pressure and suction sides. On the suction side, the Nusselt number for the $0.9 \%$ tip clearance dropped more significantly at $\mathrm{s} / \mathrm{C}=0.29$. Beyond $\mathrm{s} / \mathrm{C}=0.73$, after the overall peak heat transfer, the $1.8 \%$ tip gap case showed about a $23 \%$ drop in Nusselt number. This drop could be a result of weakened suction side leakage vortex with a larger tip gap. The pressure side heat transfer did not show a marked difference except that two gages were lost for the $1.8 \%$ tip gap case. 


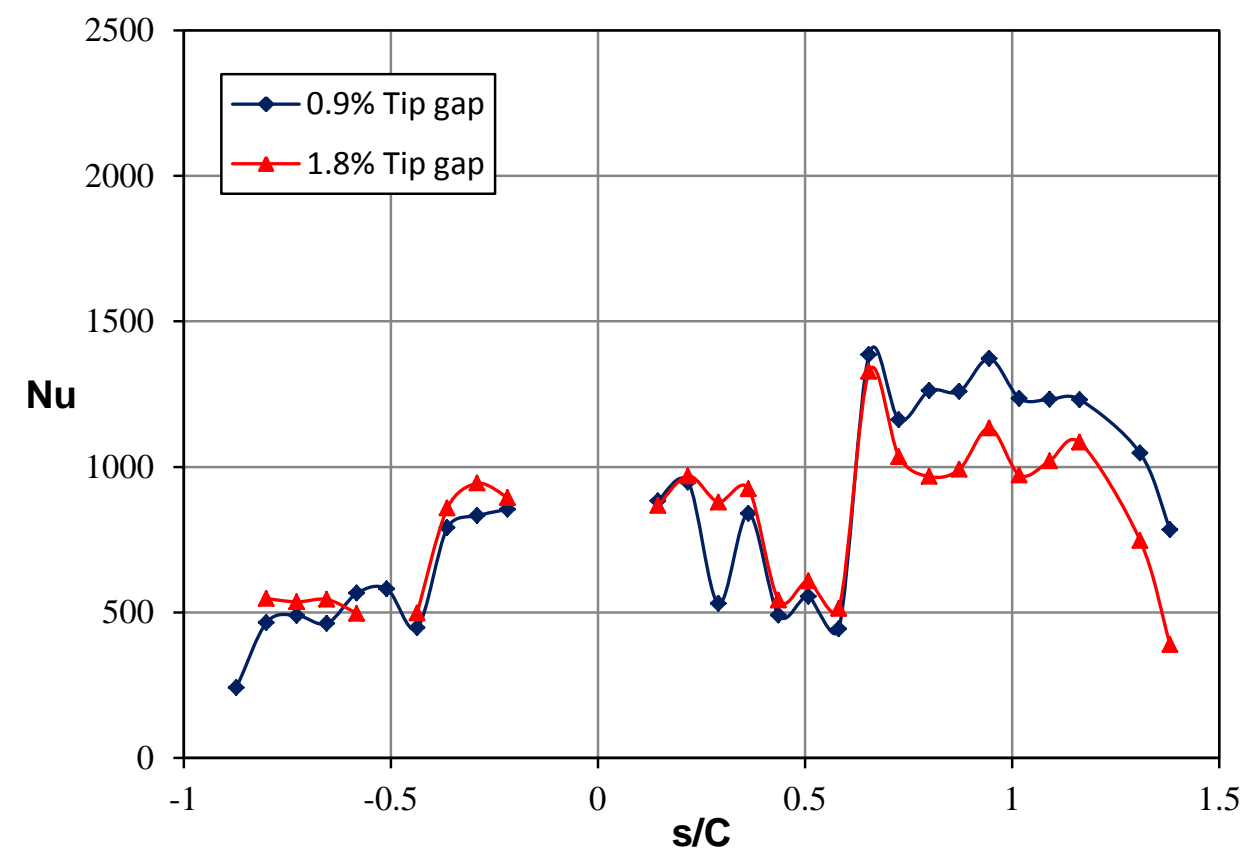

Figure E. 5 Heat transfer distribution at exit Mach number 0.7

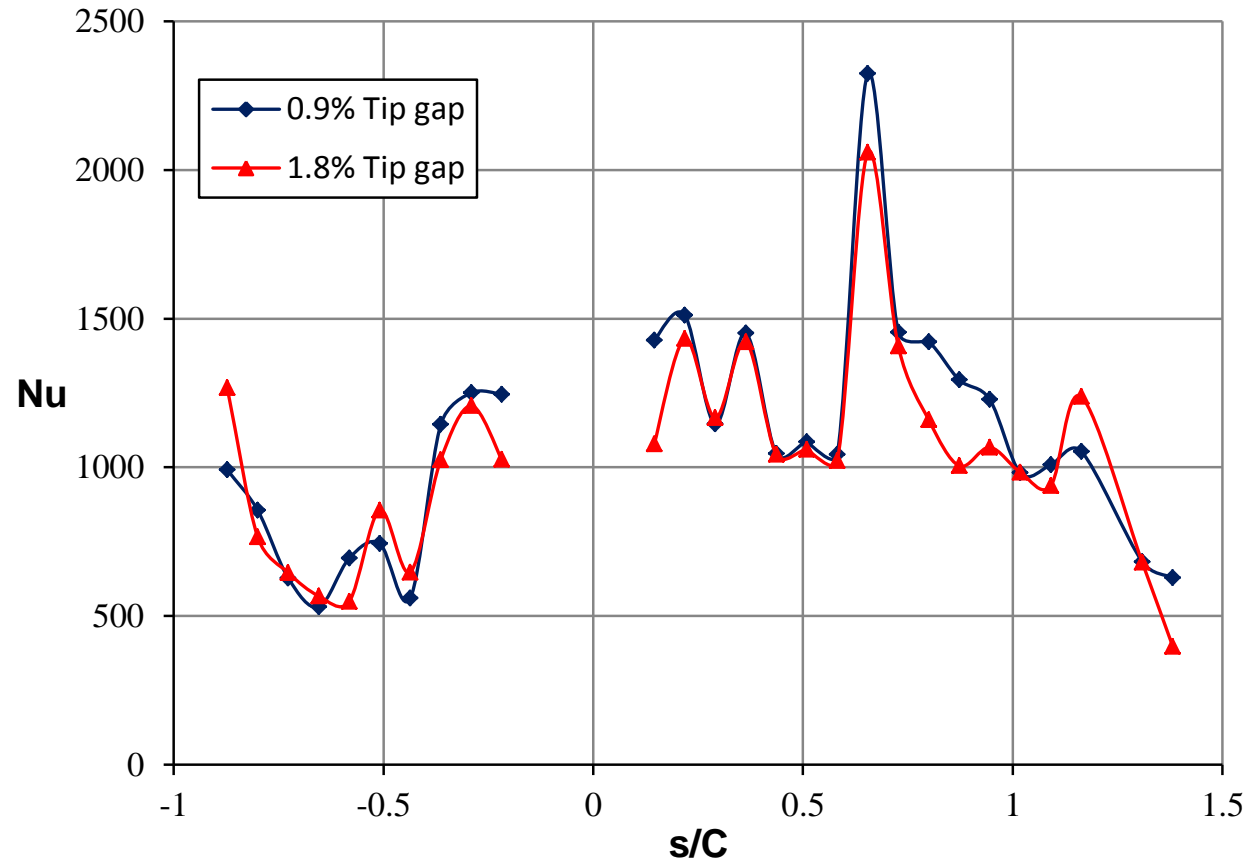

Figure E. 6 Heat transfer distribution at exit Mach number 1.05

At 1.05 exit Mach, as shown in Figure E.6, the heat transfer distribution for both tip gap cases appeared similar. The peak Nusselt number at $\mathrm{s} / \mathrm{C}=0.66$ is $13 \%$ higher for the $0.9 \%$ tip gap 
case compared to the $1.8 \%$ tip clearance test. Beyond $\mathrm{s} / \mathrm{C}=0.73$, the Nusselt number in both cases decreased although that for the higher tip gap case decreased faster. A local peak was observed at $\mathrm{s} / \mathrm{C}=1.16$ for both cases and then a decrease in Nusselt number occurred afterwards. The pressure side Nusselt number distributions were similar for both cases.

\section{Exit Mach number Effects on Heat Transfer Distribution}

The comparison between all three exit Mach number test conditions for a tip clearance of $1.8 \%$ is shown in Figure E.7. Similar to the results at $0.9 \%$ percent tip gap, the Nusselt number distribution for all three cases in Figure E.7 showed a similar pattern.

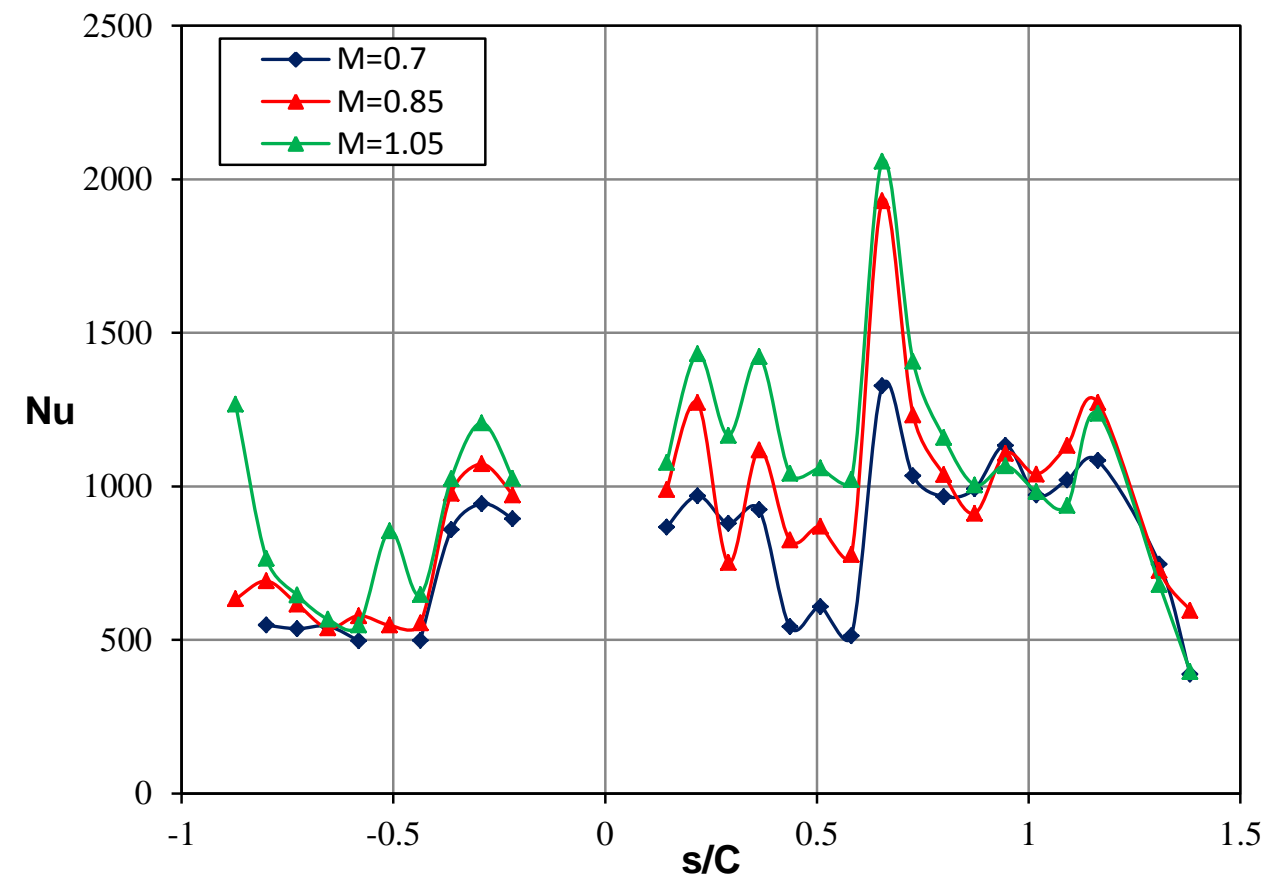

Figure E. 7 Heat transfer distribution for $1.8 \%$ tip clearance

Nusselt number was augmented by $27 \%$ at $\mathrm{s} / \mathrm{C}=0.36$ for the 1.05 exit Mach case relative to the results of the 0.85 exit Mach case. A $17 \%$ drop however was found at the same location for the 0.7 exit Mach test. Further downstream on the suction side, the overall peak levels of heat transfer for all three cases occurred at an s/C location of 0.66 . At this location, only a $7 \%$ rise in Nusselt number was observed for the higher Mach number case. For the lower Mach number case, a much significant Nusselt number drop of $31 \%$ was obtained. Relatively steady heat transfer levels were seen between $0.8<\mathrm{s} / \mathrm{C} \leq 1.2$ for all three cases before a sharp decline at s/C $>1.2$ took place. On the pressure side, similar results were also achieved in relation to the $0.9 \%$ tip gap case. A local 
peak in heat transfer still existed for the 1.05 exit Mach case at $\mathrm{s} / \mathrm{C}=-0.5$. No peak was observed for the 0.85 exit Mach test, which could mean that the pressure differential between the suction and pressure sides at this near-midchord location was not the largest. 


\section{Appendix F Uncertainty Analysis}

An uncertainty analysis was performed on the heat transfer calculations to determine the total uncertainty on the Nusselt numbers measured by each thin film gauge, and for the heat transfer coefficients for the IR technique. The total uncertainty consists of a bias error and a precision error. The bias error is the error that is constant for a set of measurements. Typically, bias errors are the result of a calibration error. The bias error was determined using the perturbation method described by Moffat [G-8]. This method takes into account the error associated with the measurement and tracks the error as it propagates through calculations. For the thin film gages, the bias error for each experiment was calculated to be $8.5 \%$ with the majority of the error being associated to the uncertainties of the material properties of the Macor. Each gauge had the same bias error because the same gauges and data reduction technique was used on each one.

The precision error is the error that is due to the run to run repeatability of the measurements. The precision error was calculated using a statistical method provided in equation F.1,

$$
P=\frac{t \cdot S_{N u}}{\overline{N u} \cdot \sqrt{N}}
$$

where $\mathrm{S}_{\mathrm{Nu}}$ is the standard deviation of the Nusselt number, $\mathrm{Nu}$ is the average Nusselt number, $\mathrm{N}$ is the number of runs and $t$ is the value of a two sided Students- $t$ distribution at a $90 \%$ confidence interval. The precision error varied for set of data and for each gauge. The minimum and maximum precision errors were calculated to be $0.2 \%$ and $7.8 \%$ respectively.

With the bias error and the precision error calculated, the total uncertainty could be calculated. The total uncertainty is given by equation F.2,

$$
\Delta=\sqrt{B^{2}+P^{2}}
$$

where $\mathrm{B}$ is the bias error and $\mathrm{P}$ is the precision error. The average total uncertainty for all of the near-tip heat transfer experiments ranges between 8.5 to $11.5 \%$. For the IR technique, the blade tip heat transfer coefficients were obtained with an average uncertainty of $\pm 9.5 \%$. 


\section{Appendix G References}

[G-1] Reagle, C.J., 2009, "Heat Transfer Measurements Using Thin Film Gauges and Infrared Thermography on a Film Cooled Transonic Vane," Master's Thesis, Virginia Polytechnic Institute and State University.

[G-2] Cress, R.D., 2006, "Turbine Blade Heat Transfer Measurements in a Transonic Flow Using Thin film Gages," Master's Thesis, Virginia Polytechnic Institute and State University.

[G-3] Smith, D.E., Bubb, J.V., Popp, O., Grabowski, H.C., Diller, T.E. Schetz, J.A. and Ng. W.F., 2000, "An Investigation of Heat Transfer in a Film Cooled Transonic Turbine Cascade, Part I: Steady Heat Transfer,” ASME GT-2000-202.

[G-4] Popp, O., Smith, D.E., Bubb, J.V., Grabowski, H.C., Diller, T.E. Schetz, J.A. and Ng. W.F., 2000, "An Investigation of Heat Transfer in a Film Cooled Transonic Turbine Cascade, Part II: Unsteady Heat Transfer,” ASME GT-2000-203.

[G-5] Nasir, S., Carullo, J.S., Ng, W.F., Thole, K.A., Wu, H., Zhang, L.J., and Moon, H.K., 2009, "Effects of Large Scale High Freestream Turbulence, and Exit Reynolds Number on Turbine Vane Heat Transfer in a Transonic Cascade," ASME J. Turbomach., 131, 021021

[G-6] Carullo, J.S., 2006, "Effects of Freestream Turbulence, Turbulence Length Scale, and Reynolds Number on Turbine Blade Heat Transfer in a Transonic Cascade," Master's Thesis, Virginia Polytechnic Institute and State University.

[G-7] Nasir, S., 2008, "Showerhead Film Cooling Performance of a Turbine Vane at High Freestream Turbulence in a Transonic Cascade," PhD Dissertation, Virginia Polytechnic Institute and State University.

[G-8] Moffat, R. J., 1988, "Describing Uncertainties in Experimental Results," Exp. Thermal and Fluid Science, 1988, pp. 3-17 